\title{
The Late Endosomal Pathway Regulates the Ciliary Targeting of Tetraspanin Protein Peripherin 2
}

\author{
DWataru Otsu, ${ }^{1}$ Ya-Chu Hsu, ${ }^{1}$ Jen-Zen Chuang, ${ }^{1}$ and ${ }^{\circledR C}$ Ching-Hwa Sung ${ }^{1,2,3}$ \\ Departments of ${ }^{1}$ Ophthalmology, ${ }^{2}$ Cell and Developmental Biology, Weill Cornell Medicine, New York, New York 10065, and ${ }^{3}$ Infectious Disease and Cancer \\ Research, Kaohsiung Medical University, Kaohsiung City 807, Taiwan
}

Peripherin 2 (PRPH2) is a tetraspanin protein concentrated in the light-sensing cilium (called the outer segment) of the vertebrate photoreceptor. The mechanism underlying the ciliary targeting of PRPH2 and the etiology of cone dystrophy caused by PRPH2 mutations remain elusive. Here we show that the late endosome (LE) is the main waystation that critically sorts newly synthesized PRPH2 to the cilium. PRPH2 is expressed in the luminal membrane of the LE. We delineate multiple C-terminal motifs of PRPH2 that distinctively regulate its LE and ciliary targeting through ubiquitination and binding to ESCRT (Endosomal Sorting Complexes Required for Transport) component Hrs. Using the newly developed TetOn-inducible system in transfected male and female mouse cones in vivo, we show that the entry of nascent PRPH2 into the cone outer segment can be blocked by either cone dystrophy-causing C-terminal mutations of PRPH2, or by short-term perturbation of the LE or recycling endosomal traffic. These findings open new avenues of research to explore the biological role of the LE in the biosynthetic pathway and the etiology of cone dystrophy caused by PRPH2 mutations and/or malfunctions of the LE.

Key words: cone photoreceptor; late endosome; macular degeneration; outer segment; peripherin 2/rds; primary cilium

\section{Significance Statement}

Peripherin 2 (PRPH2) is a tetraspanin protein abundantly expressed in the light-sensing cilium, the outer segment, of the vertebrate photoreceptor. The mechanism underlying the ciliary transport of PRPH2 is unclear. The present study reveals a novel ciliary targeting pathway, in which the newly synthesized PRPH2 is first targeted to the lumen of the late endosome (LE) en route to the cilia. We deciphered the protein motifs and the machinery that regulates the LE trafficking of PRPH2. Using a novel TetOninducible system in transfected mouse cones, we showed that the LE pathway of PRPH2 is critical for its outer segment expression. A cone dystrophy-causing mutation impairs the LE and ciliary targeting of PRPH2, implicating the relevance of LE to cone/ macular degenerative diseases.

\section{Introduction}

The endosomal system is where the endocytic and exocytic pathways intersect. The endosomal system consists of a mosaic of

\footnotetext{
Received 0ct. 31, 2018; revised Jan. 18, 2019; accepted Feb. 10, 2019.

Author contributions: W.O., J.-Z.C., and C.-H.S. designed research; W.O., Y.-C.H., and J.-Z.C. performed research; W.O., Y.-C.H., J.-Z.C., and C.-H.S. analyzed data; C.-H.S. wrote the paper.

This work is supported by grants from the National Institutes of Health (NIH; Grants R01-EY-016805 and EY029428), Kaohsiung Medical University Research Foundation (Grant 105KMUOR02), and the Bohmfalk Charitable Trust. C.-H.S. is a recipient of a Stein Innovation Award from Research To Prevent Blindness and an Alcon Research Award. Focused-ion bean scanning electron microscopy was performed at the Simons Electron Microscopy Center and National Resource for Automated Molecular Microscopy located at the New York Structural Biology Center (New York, NY), which was supported by grants from the Simons Foundation (Grant SF349247), NYSTAR (Empire State Development's Division of Science, Technology and Innovation), and the NIH National Institute of General Medical Sciences (Grant GM-103310) with additional support from the NIH (Grant RR-0293T00). We thank Drs. R.S. (The University of British Columbia,Vancouver, BC, Canada), E. Rodriguez-Boulan (Weill Cornell Medical College, New York, NY), T.-S. Jou (Stanford University School of Medicine, Stanford, California), E.L. Snapp (Albert Einstein College of Medicine, Bronx, NY), C.L.Cepko (Harvard Medical School, Boston, MA), F. Sanchez-Madrid (Hospital Universitario de la Princesa, Madrid, Spain), S. Urbé (University of Liverpool, Liverpool, UK), M.R. Al-Ubaidi (University of Houston, Houston, TX), and T.E. McGraw (Weill Cornell Medical College, New York, NY) for providing valuable reagents; and
}

interconnected compartments, including early endosomes, recycling endosomes, and late endosomes (LE). A subset of LEs can further mature to form lysosomes (Lys). Both LE and Lys have an acidic lumen and an overlapping protein expression pattern [e.g., Lamp (lysosomal-associated membrane protein) 1, Lamp2, CD63]. The main function of the Lys is protein degradation, whereas LE (or multivesicular bodies) has emerged as an important sorting station. Membrane cargoes are recruited into the lumen of the LE as intraluminal vesicles through Endosomal Sorting Complexes Required for Transport (ESCRT)-dependent or ESCRT-independent pathways (Piper and Katzmann, 2007).

The Eye-Bank for Sight Restoration Inc. for human donor eyes. We also thank Emily Benson (Renovo Neural, Cleveland, $\mathrm{OH}$ ) for processing/imaging collection for serial block face scanning electron microscopy.

The authors declare no competing financial interests.

Correspondence should be addressed to Jen-Zen Chuang at jzchuang@med.cornell.edu or Ching-Hwa Sung at chsung@med.cornell.edu.

https://doi.org/10.1523/JNEUROSCI.2811-18.2019

Copyright $\odot 2019$ the authors 
LE luminal membrane components have been shown to recycle back to the cell surface or trans-Golgi network (TGN), or be released into the extracellular exosomes upon LE fusion to the plasma membrane (Kobayashi et al., 2000; Kleijmeer et al., 2001; Abrami et al., 2004; Le Blanc et al., 2005). Other membrane destinations of the LE luminal cargoes and the mechanisms underlying LE trafficking are incompletely understood.

The tetraspanins are a family of four-pass transmembrane proteins that have both their $\mathrm{N}$ and $\mathrm{C}$ termini facing the cytosol. Tetraspanins are known to act as molecular scaffolds and have been implicated in several pathological conditions (e.g., cancer, infectious disease, inflammation, blindness; Hashimoto et al., 2017). Peripherin 2 (PRPH2 or peripherin/rds) is a photoreceptor-specific tetraspanin protein abundantly expressed in the cilium (outer segment) of both rods and cones. The outer segments contain disc membranes that undergo constant renewal (Young, 1967). PRPH2 is essential for the morphogenesis of the outer segment. PRPH2-deficient (rds/rds) mice fail to develop outer segments and undergo subsequent retinal degeneration (Sanyal et al., 1980). To date, the mechanism that underlies the ciliary transport of $\mathrm{PRPH} 2$ remains unclear. Chimera mapping studies performed in transgenic frog rods and transfected RPE-1 cells showed that the cytoplasmic C-terminal tail is required for the ciliary targeting of PRPH2 (Tam et al., 2004; Salinas et al., 2013; Tian et al., 2014). Ectopically expressed PRPH2 in AD293T and COS cells was absent from the plasma membrane and, instead, was distributed on internal membranes of unknown nature (Loewen et al., 2003; Conley et al., 2010; Khattree et al., 2013; Milstein et al., 2017; Salinas et al., 2017). Endoglycosidase sensitivity assays indicate that PRPH2 does not undertake the canonical Golgi-mediated secretory pathway (Connell and Molday, 1990; Tian et al., 2014).

PRPH2 has several proposed functions, including membrane fusion, membrane curvature formation/tubulation, and ciliary shedding (Boesze-Battaglia et al., 1998; Molday et al., 2004; Goldberg, 2006; Khattree et al., 2013; Hsu et al., 2015; Stuck et al., 2016; Salinas et al., 2017). A large number of human mutations of the $P R P H 2$ gene have been linked to a spectrum of hereditary retinal degenerative diseases, affecting predominantly either rods or cones (Stuck et al., 2016). The majority of PRPH2 mutations linked to rod dystrophy cause impaired protein stability/oligomerization and endoplasmic reticulum (ER) exit (Loewen et al., 2003; Conley et al., 2010). The cellular defect caused by cone dystrophy-associated PRPH2 mutants is unknown.

Using two ciliated cell models and mouse cones in vivo, the present work shows that, unexpectedly, the nascent PRPH2 is mainly held in the lumen of the LE en route to the cilia. We mechanistically dissected the C-terminal sorting motifs of PRPH2, which are differentially required for ubiquitination, the interaction with ESCRT component Hrs, and the trafficking through the LE for ciliary entry. We provide both morphological and functional evidence supporting the importance of endosomal trafficking in the outer segment targeting of PRPH2 in mouse cones. These studies were aided by $3 \mathrm{D}$ electron microscopy (EM) technology and our newly established TetOn-regulated gene manipulation in transfected mouse cones. These results reveal a novel ciliary targeting pathway and open a new direction for investigating the functions of LE in cones and the relevance of LEs to cone degenerative diseases.

\section{Materials and Methods}

Reagents. All chemicals were obtained from Sigma-Aldrich unless otherwise mentioned. The following primary antibodies were used in this study: PRPH2 [1:30 for immunofluorescent (IF) assay, 1:150 for immu- noblotting (IB) assay; mouse, 3B6, a gift from Dr. R.S. Molday (Molday et al., 1987) mouse, 2H5, a gift from Dr. R.S. Molday (Connell et al., 1991); 1:30 for IF assay, 1:200 for IB assay, 1:100 for immunoprecipitation (IP) assay; rabbit, catalog \#18109-1-AP, Proteintech (RRID:AB_10665364); 1:100 for IF assay, 1:1000 for IB); FLAG M2 (1:1000 for IF assay; mouse, catalog \#P2983, Sigma-Aldrich; RRID:AB_439685); 1:2000 for IF, 1:20,000 for IB, GFP [chicken, catalog \#GFP-1020, Aves Labs (RRID: AB_10000240); $1 \mu \mathrm{g}$ for IP assay, mouse, catalog \#A-11120, Thermo Fisher Scientific (RRID:AB_221568)]; mCherry (mCh; 1:2000 for IF assay, 1:20,000 for IB assay; goat; catalog \#AB0040-200, SICGEN; RRID: AB_2333092); DsRed (1:500 for IF assay, 1:1000 for IB assay; rabbit; catalog \#632496, Clontech Laboratories; RRID:AB_10013483); $\beta$ tubulin (1:5000 for IB assay; mouse; catalog \#T5201, Sigma-Aldrich; RRID:AB_609915); acetylated- $\alpha$-tubulin (1:2000 for IF assay; mouse; catalog \#T6793, Sigma-Aldrich; RRID:AB_477585); detyrosinated tubulin (1:400 for IF assay; rabbit; catalog \#AB3201, Millipore; RRID: AB_177350); E-cadherin (1:500 for IF assay; rat; catalog \#U3254, SigmaAldrich; RRID:AB_477600); gp135 (1:200 for IF assay; rabbit; a gift from Dr. T.S. Jou; Jou and Nelson, 1998); canine Lamp2 (1:100 for IF assay; mouse; AC17, a gift from Dr. E. Rodriguez-Boulan; Nabi et al., 1991); Rab11a (1:200 for IF assay; rabbit catalog \#71-5300, Thermo Fisher Scientific; RRID:AB_2533987); Rab11b (1:200 for IF assay, 1:500 for IB assay; rabbit; catalog \#19742-1-AP, Proteintech; RRID:AB_10642010); GM130 (1:200 for IF assay; mouse; catalog \#610822, BD Biosciences; RRID:AB_398141); EEA1 (1:400 for IF; mouse; catalog \#610456, BD Biosciences; RRID:AB_397829); Lamp1 [1:10 for IF, mouse, catalog \#H4A3, Developmental Studies Hybridoma Bank (RRID:AB_2296838); 1:50 for IF assay, rat, catalog \#1d4b, Developmental Studies Hybridoma Bank (RRID:AB_2134500)]; TGN38 (1:1000 for IF assay; rabbit; WMC18, a gift from Dr. E. Rodriguez-Boulan; Gravotta et al., 2012); Hrs (1:200 for IB; mouse; catalog \#sc-271455, Santa Cruz Biotechnology; RRID:AB_10648901); ubiquitin (1:200 for IB assay; mouse, catalog \#sc8017, Santa Cruz Biotechnology; RRID:AB_628423); and cone opsin (1: 100,000 , used as a cocktail for the detection of all cones, IF assay; rabbit, S-cone opsin for JH455 and M-cone opsin for JH492, gifts from Dr. J. Nathans; Wang et al., 1992). Alexa Fluor dye-conjugated secondary antibodies were obtained from Thermo Fisher Scientific or Jackson ImmunoResearch.

All the plasmids made in this study were generated by standard molecular cloning methods. The coding regions obtained by PCR were all sequence confirmed. The human PRPH2 gene was amplified from a human retina cDNA library (gift from Jeremy Nathans, Johns Hopkins University School of Medicine, Baltimore, MD). The 3'-untranslated region of the human $P R P H 2$ gene was $\mathrm{PCR}$ synthesized to mimic the $\mathrm{C}$ terminus of the 1137TG mutant. TetOn-PRPH2, TetOn-GFP-HrsshRNA, TetOn-GFP-Rab11b-shRNA, or TetOn-control short hairpin RNA (shRNA) plasmids were generated by inserting the sequences of tetracycline operator-miniCMV promoter and rtTA3 (from the TRIPZ Lentiviral vector; Thermo Fisher Scientific) into the pCAG vector containing either PRPH2 cDNA or Hrs-shRNA, or Rab11-shRNAs. The miR-E backbone sequence was inserted with the targeting sequences of Hrs-shRNA\#1 (ID Hgs.1087), Hrs-shRNA\#2 (ID Hgs.352), and Rab11bshRNA (ID Rab11b.236) were obtained from Chang et al. (2006, their supplemental Table S3). The scrambled control shRNA sequence was AATGACGACCACGAGGAATGAG-3'. All constructs involving PCR were confirmed by sequencing. The following plasmids have been previously reported. The plasmid encoding bovine PRPH2 was a gift from Dr. R.S. Molday (Goldberg et al., 1995). PRPH2-GFP, PRPH2-mCh, and CAG:LoxP-neo-LoxP-PRPH2-GFP were made in our laboratory (Hsu et al., 2015). The following mammalian expression vectors for fluorescence tagged reporters were used: EYFP-GalT [J. Lippincott-Schwartz (Cole et al., 1996)/catalog \#11936, Addgene]; GFP-EEA1 [S. Corvera (Lawe et al., 2000)/catalog \#42307, Addgene]; Lamp1-GFP [E. Dell'Angelicaå (Falcón-Pérez et al., 2005)/catalog \#34831, Addgene]; GFP-Sec61beta (T. Rapoport/catalog \#15108 Addgene); and RFP-Hrs [E. De Robertis (Taelman et al., 2010)/catalog \#29685, Addgene]. GFP-CD63 was a gift from Dr. F. Sanchez-Madrid (Mittelbrunn et al., 2011). GFP-Rab1 la was a gift from Dr. T. McGraw (Thuenauer et al., 2014). GFP-Hrs was a gift from Dr. S. Urbé (Urbé et al., 2003). Plasmid coding superfolder GFP was a gift 
A

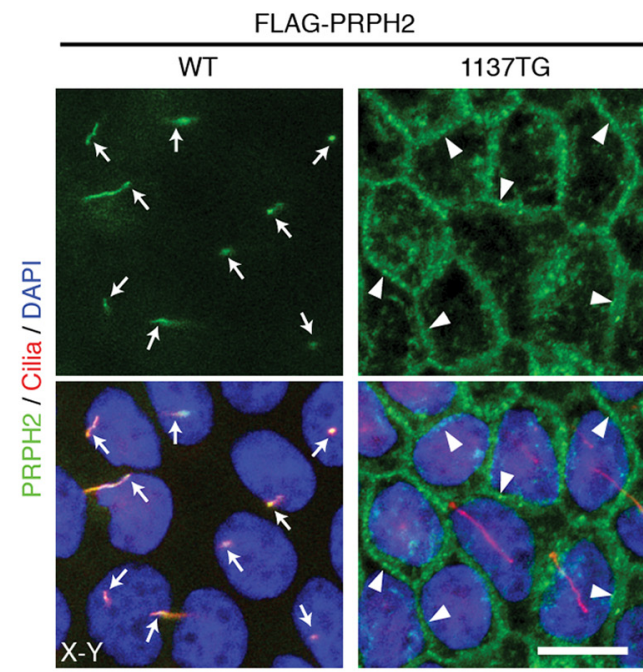

B

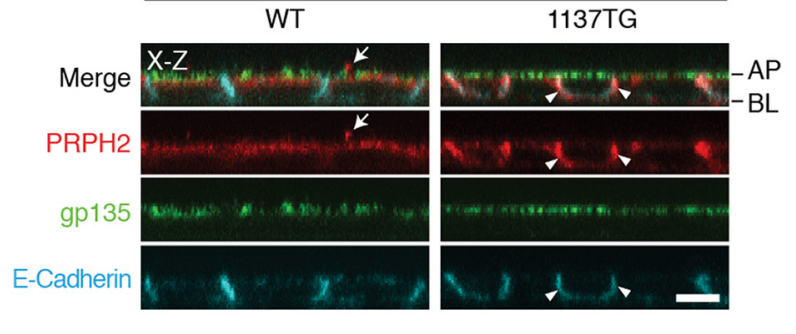

C

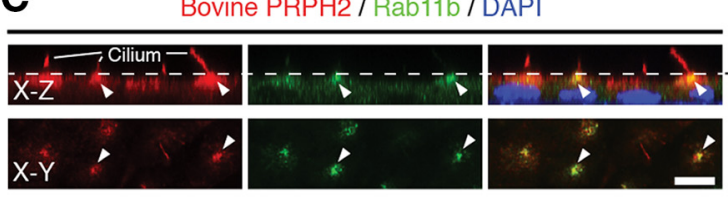

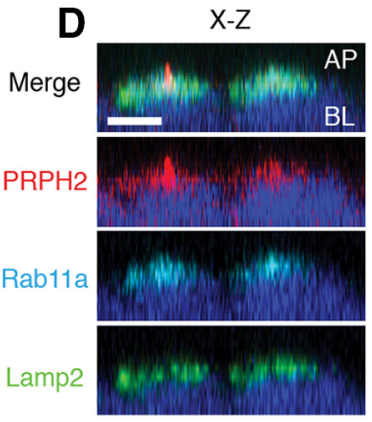

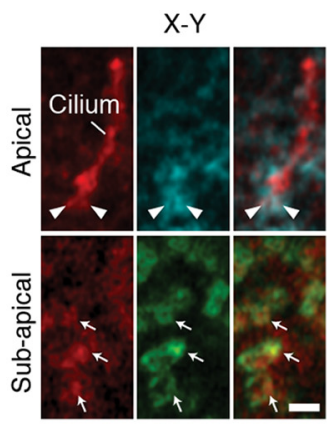

E
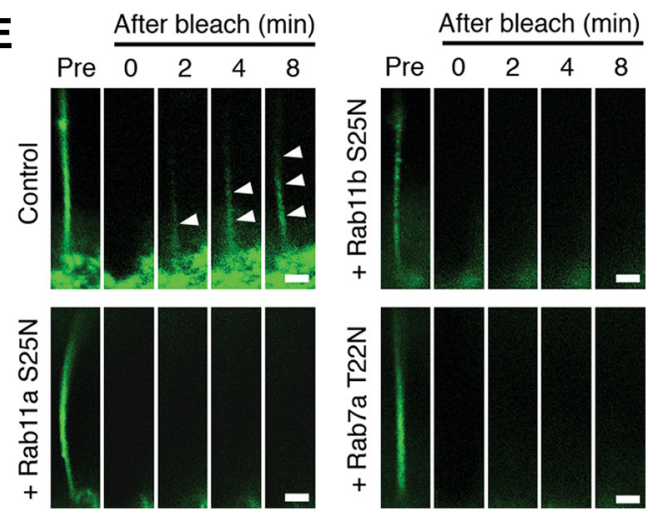

$\mathbf{F}$

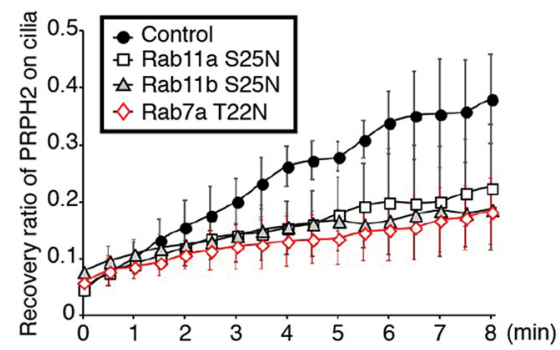

Figure 1. Ciliary expression of PRPH2 requires its C terminus and proper endosomal trafficking. $A$, WT and 1137TG FLAG-PRPH2 expressed in MDCK cell lines were stained for FLAG and a cilium marker (detyrosilated tubulin). Optical sections horizontal to the monolayers ( $x$-y plane) show the ciliary expression of the WT (arrows) and the cell border expression of the 1137TG mutant (arrowheads). B, MDCK cells stably expressing WT or 1137TG PRPH2-mCh were stained for mCh, gp135, and E-cadherin. Optical sections perpendicular to the monolayer ( $x-z$ plane) show the WT being expressed on the cilium (arrows) and the 1137TG mutant being expressed on the lateral membrane (arrowheads). AP, Apical; BL, basolateral. C, Representative $x-z$ and $x-y$ confocal sections show that in polarized MDCK monolayers, the stably expressed nontagged WT bovine PRPH2 (labeled by 3 B6 antibody) is localized on the cilia extending from the apical surface (dashed lines). PRPH2 is also enriched in the Rab11b-labeled recycling endosomes at the subapical/periciliary region (arrowheads). $\boldsymbol{D}$, The $x-z$ (left) and $x-y$ (right) sectioning planes of confocal images (with Airyscan head) show the spatial relationship among Rab11a (arrowheads), Lamp2 (arrows), and stably expressed PRPH2-mCh. E, PRPH2-GFP expressed in the cilia was imaged before (Pre) and after photobleaching in control cells and cells transfected with indicated mCh-Rab mutants. Red channel is not shown. Arrowheads mark the PRPH2-GFP recovered in the cilia after photobleaching. $\boldsymbol{F}$, Quantification of $\boldsymbol{E}$. $y$-Axis represents the relative intensity of PRPH2-GFP recovered on cilia after photobleaching; the ciliary signal of PRPH2-GFP before bleaching is considered as 1 (mean \pm SD; $n=6, N=3)$. Blue: DAPI nuclear stain $(\boldsymbol{A}, \boldsymbol{C}, \boldsymbol{D})$. Scale bars: $\boldsymbol{A}-\boldsymbol{C}, x-z$ in $\boldsymbol{D}, 10 \mu \mathrm{m} ; x-y$ in $\boldsymbol{D}, \boldsymbol{E}, 1 \mu \mathrm{m}$.

from Dr. E.L. Snapp (Aronson et al., 2011). ER ${ }^{\mathrm{T} 2}-\mathrm{Cre}-\mathrm{ER}^{\mathrm{T} 2}$ was a gift from Dr. C. Cepko (Matsuda and Cepko, 2007).

Cell culture studies: transfection, staining, and imaging. 293T cells (catalog \#CRL-3216, ATCC; RRID:CVCL_0063) were transfected using a polyethylenimine-based method. Madin-Darby canine kidney (MDCK) cells (catalog \#CCL-34, ATCC; RRID:CVCL_0422) were transfected using the Amaxa Nucleofector System (Lonza) or Lipofectamine 2000 (Thermo Fisher Scientific). MDCK stable clones were generated using G418 selection and were selected based on both immunofluorescent and immunoblotting assays. To generate polarized ciliated MDCK cells, the cells were plated on Transwell filters (Corning) at a density of $1 \times 10^{5}$ cells/6.5 mm dish for 3-4 d. 661W cells [a gift from M.R. Al-Ubaidi (Al-Ubaidi et al., 2008); RRID:CVCL_6240) were transfected using the Amaxa Nucleofector System, and ciliogenesis was induced by serum-free starvation for $48 \mathrm{~h}$ (at a plating density of $5 \times 10^{5} / 35 \mathrm{~mm}$ dish). For the pulse-chase experiments, $4 \mathrm{~h}$ post-transfection $661 \mathrm{~W}$ cells were incubated at $15^{\circ} \mathrm{C}$ for $2 \mathrm{~h}$ and then transferred back to $37^{\circ} \mathrm{C}$ for the indicated time. Cycloheximide $(100 \mu \mathrm{g} / \mathrm{ml})$ was added in the culture media during the last $30 \mathrm{~min}$ of $15^{\circ} \mathrm{C}$ incubation and throughout the chase. In some experiments, brefeldin A $(0.5 \mu \mathrm{g} / \mathrm{ml})$ was also added during the chase. $661 \mathrm{~W}$ stable cell lines were generated as described for MDCK cells.

Immunostaining and imaging of cultured cells. For immunostaining, the cells were fixed with $4 \%$ paraformaldehyde (PFA) in PBS-C/M (PBS containing $2 \mathrm{mM} \mathrm{MgCl}_{2}$ and $0.2 \mathrm{mM} \mathrm{CaCl}_{2}$ ) for $10 \mathrm{~min}$. After quenching with $50 \mathrm{mM} \mathrm{NH}_{4} \mathrm{Cl}$, the cells were permeabilized and blocked with the PBTAD buffer (PBS-C/M plus $0.25 \%$ Triton X-100, 0.5\% bovine serum albumin (BSA), $0.2 \mathrm{mg} / \mathrm{ml} \mathrm{Na-azide,} \mathrm{and} 0.3 \mathrm{~mm}$ DAPI) followed by primary and secondary antibodies. For staining involving Lamp2, PBS$\mathrm{C} / \mathrm{M}$ supplemented with $0.5 \%$ saponin, $2 \%$ bovine serum albumin, and $0.3 \mathrm{~mm}$ DAPI for $30 \mathrm{~min}$ was used for the cell permeabilization/blocking instead. Confocal images of stained cells were acquired by a $63 \times$ objective on a Zeiss LSM880 microscope; $0.24-\mu \mathrm{m}$-thick single sections are shown for all images acquired from the subconfluent cells. To obtain super-resolution-grade images, the Airyscan system on the Zeiss LSM880 microscope was used. Images were processed using Photoshop CS6 (Adobe Systems) for presentation. Some images were edited with the 
A
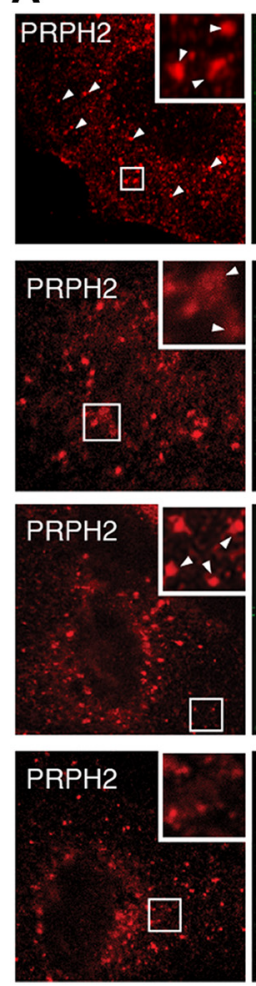
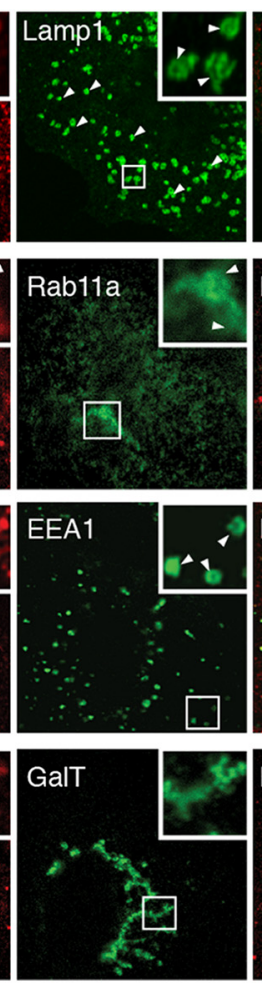

B
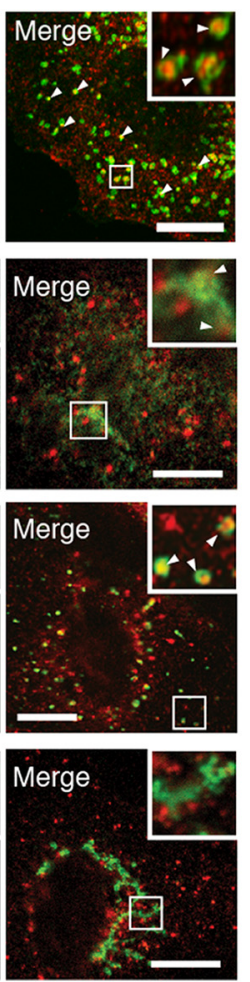

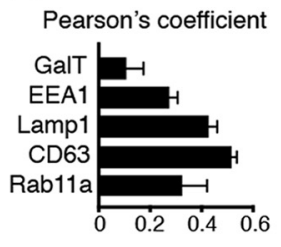

C PRPH2-mCh
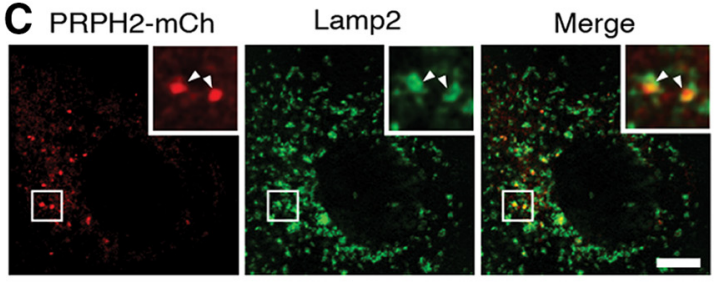

D $\mathrm{PRPH} 2-\mathrm{mCh}$

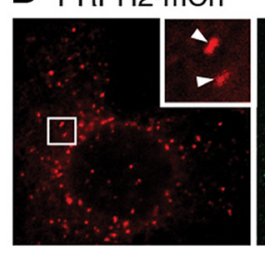

Lamp1-GFP

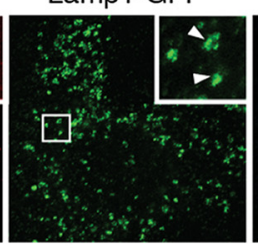

GM130
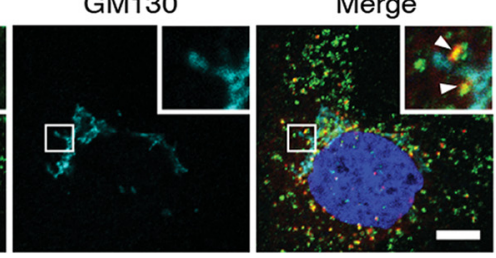

E

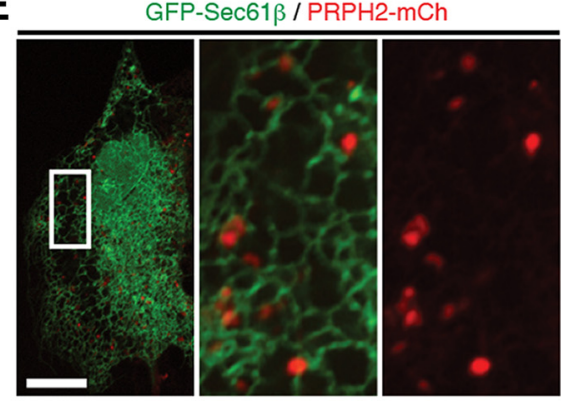

Figure 2. PRPH2 is enriched in the endosomal compartments in MDCK cells. $A$, Nontagged bovine PRPH2 was stably expressed in MDCK cells, and the cells were costained with anti-PRPH2 (3B6) and various transfected organelle-specific GFP reporters. Insets show enlarged views of the boxed areas. Arrowheads point to the overlapping PRPH 2 and GFP signals. $\boldsymbol{B}$, Quantification of $A$. Pearson's coefficients represent the extent of the colocalization between PRPH2 and the indicated organelle-specific markers (mean $\pm \mathrm{SD} ; n=36$ cells; $N=3$ ). $C-E$, Stably expressed PRPH2-mCh (detected by mCh antibody) was colabeled with Lamp2 (C), GM130 and transfected Lamp1-GFP (D), or transfected GFP-Sec61 $\beta$ (E). The boxed areas were zoomed in and further acquired by super-resolution Airyscan. Arrowheads point to signals of PRPH2-mCh overlapped with either endogenous Lamp2 (C) or Lamp1-GFP (D), but not GM130. Scale bars: $\boldsymbol{A}, \boldsymbol{C}, \boldsymbol{D}, \boldsymbol{E}, 10 \mu \mathrm{m}$.

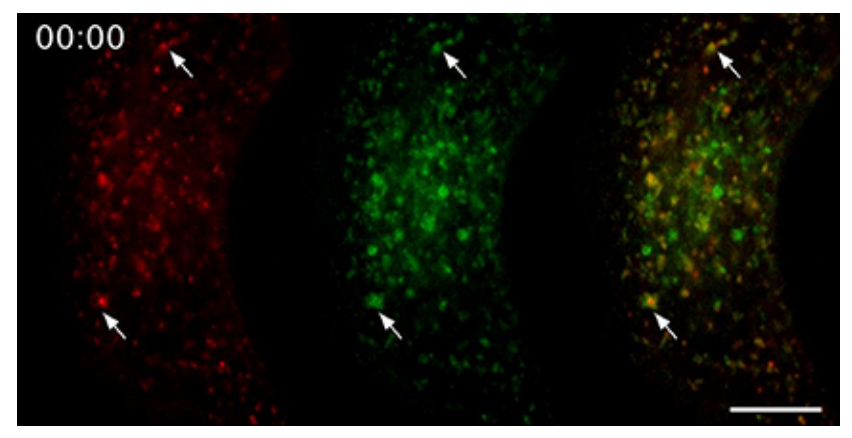

Movie 1. Time-lapse video recording of stably expressed WT PRPH2$\mathrm{mCh}$ (red) and transiently transfected Lamp1-GFP (green). Arrows point to two examples of LE that are positive for both $\mathrm{mCh}$ and GFP. Scale bar, $10 \mu \mathrm{m}$.

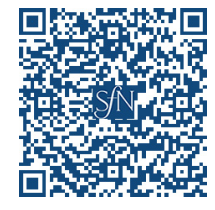

ImageJ Deconvolution plugin (DeconvolutionLab) using the TikhonovMiller algorithm. Pearson's coefficients were measured by ImageJ using the Coloc 2 plugin.

For live cell recording, cells grown on glass-bottom dishes (Greiner Bio-One) were imaged in warm recording buffer ( $140 \mathrm{~mm} \mathrm{NaCl}, 20 \mathrm{~mm}$ HEPES, pH 7.4, $2.5 \mathrm{~mm} \mathrm{KCl,} 1.8 \mathrm{~mm} \mathrm{CaCl}_{2}, 1.0 \mathrm{~mm} \mathrm{MgCl}_{2}, 20 \mathrm{~mm}$ $\mathrm{D}$-glucose, and 1\% BSA). The images were acquired by an Axio Observer Z1 fluorescence microscope (Zeiss; equipped with a Plan-Apochromat $63 \times / 1.4$ oil-immersion objective, an AxioCam HRM camera, and $\mathrm{CO}_{2}$ Module S), or TempModule S (Zeiss), or a Zeiss LSM 880 Live Imaging System (equipped with an incubation chamber, with or without an Airyscan head).
In vivo retinal transfection and retinal immunostaining. All animal manipulations were performed in accordance with the guidelines for animal experiments at Weill Medical Cornell Institutional Animal Care and Use Committee. Neonatal retinal transfection was performed in PNO Sprague Dawley rats. A single subcutaneous injection of 4-hydroxytamoxifen (2 $\mathrm{mg} / \mathrm{ml}$ in corn oil, $0.5 \mathrm{ml} / \mathrm{rat}$ ) was used to induce the expression of the transgene regulated by the $\mathrm{iP}_{\mathrm{CAG}}$ system, as described previously (Hsu et al., 2015). Embryonic retinal transfection was performed in CD1 mice at gestational day 14.5. The uterus was extracted through an abdominal incision, and DNA (in TE buffer, $<0.5 \mu \mathrm{l}$ of $1 \mu \mathrm{g} / \mathrm{ml}$ ) was injected into the subretinal space of the embryo through a beveled micropipette. Three pulses ( $45 \mathrm{~V}, 50 \mathrm{~ms}$ each, $950 \mathrm{~ms}$ intervals) were delivered by the Tweezertrode (Platinum, $7 \mathrm{~mm}$ diameter; Harvard Apparatus) placed on the injected eye through ECM830 Square Wave Pulse generator (BTX). All DNA was prepared using Endofree kit (Qiagen). For the $\mathrm{iP}_{\mathrm{CAG}}$-inducible system, a mixture of LoxP-neo-LoxP-bPRPH2-GFP: $E^{\mathrm{T} 2}$-Cre-ER ${ }^{\mathrm{T} 2}$ plasmids (ratio 2:1) was used. For TetOn-inducible knockdown, a mixture of TetOn-PRPH2 and TetOn-GFP-Hrs-shRNA or TetOn-GFPRab11b-shRNA (ratio 1:9) was used. A single intraperitoneal injection of doxycycline ( $1 \mathrm{mg} / \mathrm{kg}$ body weight) or Dox Diet ( $2 \mathrm{~g} / \mathrm{kg}$; Bio-Serv) was used to induce the expression of the transgene regulated by the TetOnsystem. All animals were kept in $12 \mathrm{~h}$ light/dark cycles until harvest and were harvested between 9:00 A.M. and noon (12:00 P.M.). At least three mice were tested for each experimental condition. All transfected eyes were harvested by transcardial perfusion with $0.1 \mathrm{M}$ phosphate buffer (PB), $\mathrm{pH} 7.4$, containing 4\% PFA plus $3.75 \%$ acrolein, and postfixed with $4 \%$ PFA plus $0.1 \%$ glutaraldehyde in 0.1 m sodium cacodylate buffer, $\mathrm{pH}$ 7.4 , overnight. Transfected mouse retinal and (submerged fixed) human retinal pieces were embedded in 5\% low-melting agarose (Amresco), sectioned by vibratome, and then subjected to immunostaining and confocal microscopic examination, as described previously (Hsu et al., 2015). 


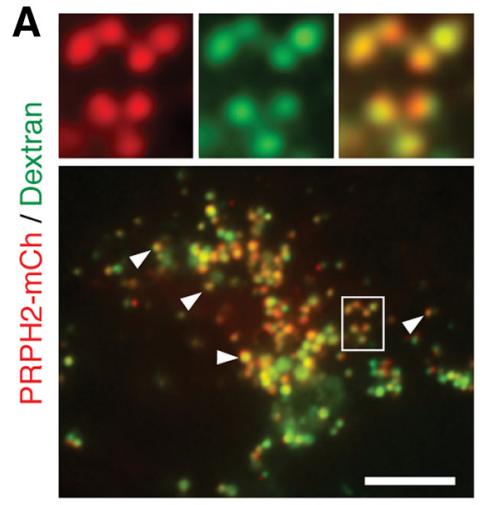

B

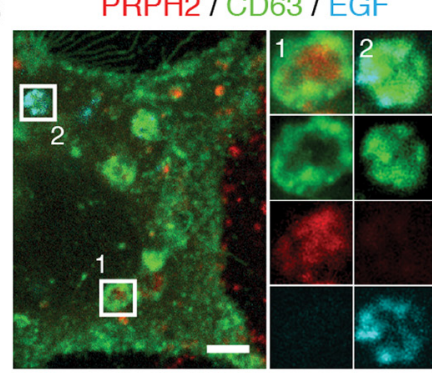

C

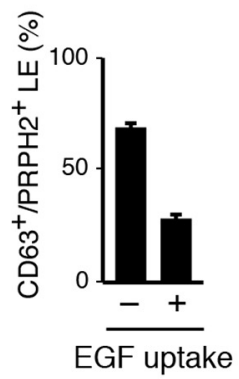

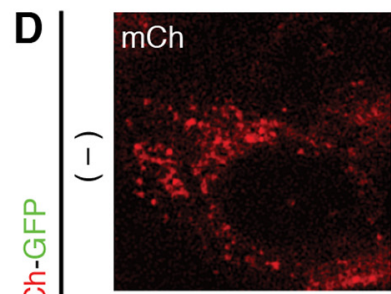
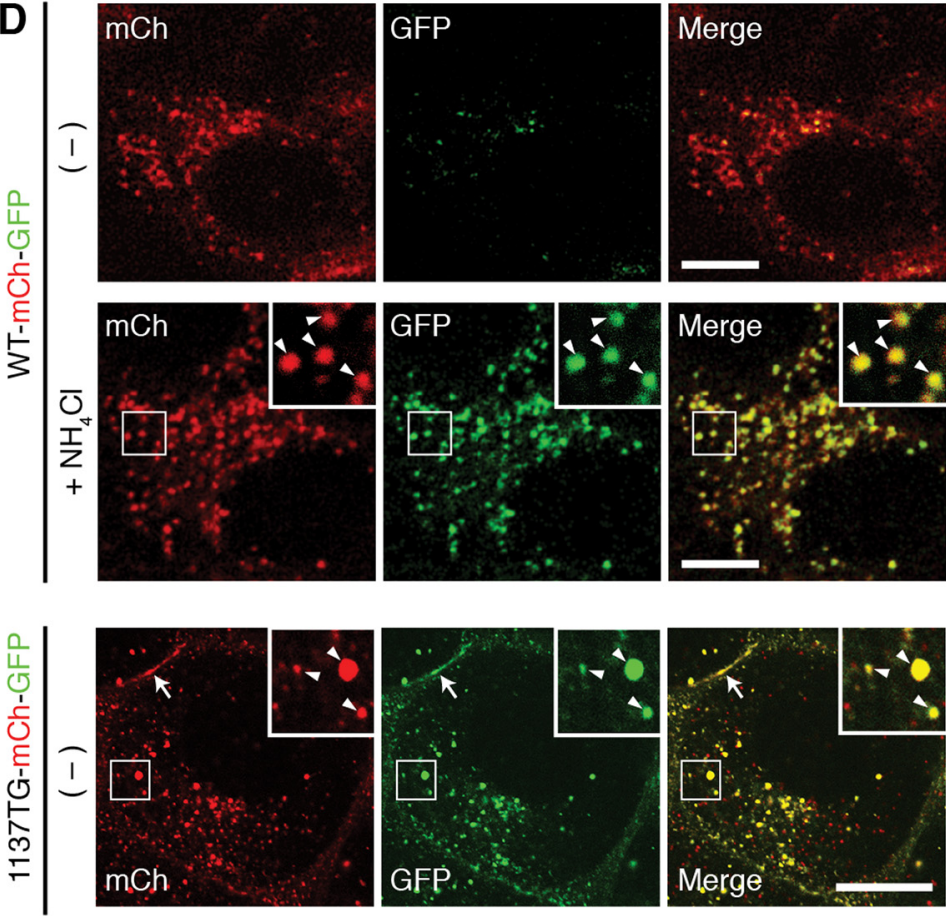

$\mathbf{F}$
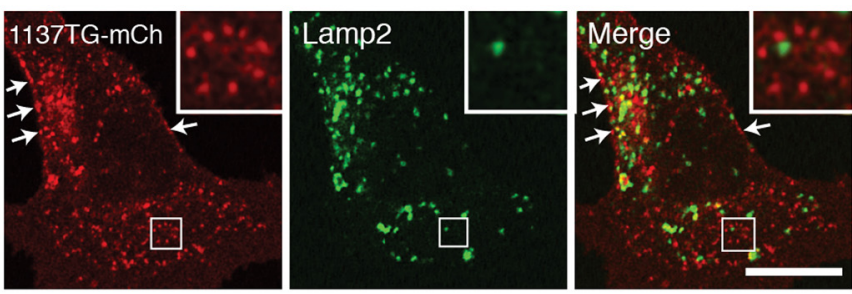

G
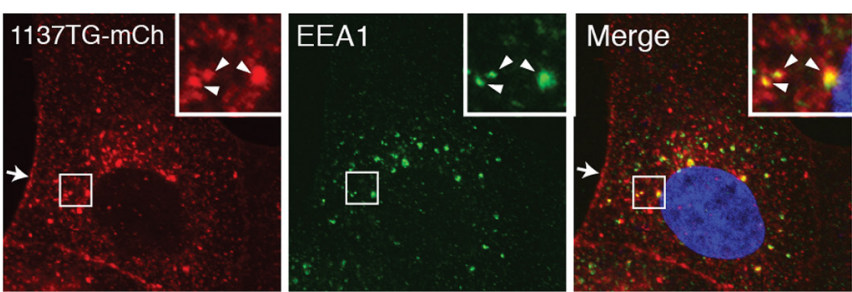

Figure 3. PRPH2 is predominantly expressed in the lumen of nondegradative LEs. A, Still images of live PRPH2-mCh expressing MDCK cells loaded with green fluorescent dextran. Low-power (bottom) and high-power (top) views of single-color and three-color merged views show many dextran-filled vacuoles that express PRPH2 (arrowheads). B, MDCK cells stably expressing nontagged bovine PRPH2 were transfected with GFP-CD63-Flag-Rab5 Q79L, and loaded with Alexa Fluor 647-conjugated EGF for 30 min. The cells were processed for staining of PRPH2 and GFP. The enlarged view of boxes 1 and 2 represent the PRPH2 ${ }^{+} / \mathrm{EGF}^{-}$and PRPH2 ${ }^{-} / \mathrm{EGF}^{+}$vacuoles, respectively. $\mathrm{C}$, Quantification of $\boldsymbol{B}$. The percentages of CD63 ${ }^{+} / \mathrm{PRPH} 2^{+}$vacuoles contain EGF $(+)$or without EGFP $\left(-\right.$ ) are shown (mean $\pm \mathrm{SD} ; n=230$ endosomes, $N=3$ ). $\mathbf{D}$, Confocal images of the direct fluorescence exhibited by the stably expressed WT PRPH2-mCh-GFP [with or without $\left(-\right.$ ] NH ${ }_{4} \mathrm{Cl}$ treatment) or 1137TG PRPH2-mCh-GFP. Arrowheads in the insets (enlarged boxed areas) point to the PRPH2-expressing vacuoles that have both red and green fluorescence. Arrows marked the cell border signal of the 1137TG mutant. $E$, Quantification of the overlap between Lamp2 and PRPH2 variants using Pearson's coefficients (mean $\pm S D ; n=41$ cells, $N=3$ ). ${ }^{* * *} p=0.00034$, Student's $t$ test. $\boldsymbol{F}, \boldsymbol{G}$, Immunostaining of Lamp2 ( $\boldsymbol{F}$; a representative image used for quantification in $\boldsymbol{E}$ ) or EEA1 $(\boldsymbol{G})$ in MDCK cells stably expressing 1137 TG PRPH2-mCh. Arrows and arrowheads point to the cell border and early endosomal expression of 1137TG, respectively. Scale bars: $\boldsymbol{A}, \boldsymbol{D}, \boldsymbol{F}, \boldsymbol{G}, 10 \mu \mathrm{m} ; \boldsymbol{B}, 2 \mu \mathrm{m}$.

Immunoelectron microscopy, transmission EM, 3D scanning EM of retinal tissues. Immunoelectron microscopy (ImmunoEM) was performed as described previously (Chuang et al., 2007). Briefly, vibratome-cut retinal slices were permeabilized in cryoprotection solution, quenched in $1 \%$ sodium borohydride, and briefly washed in $0.1 \%$ Triton X-100 containing PB (0.1 M PB) and then Tris-buffered saline (TS; $0.15 \mathrm{M} \mathrm{NaCl}$ in $0.1 \mathrm{~m}$ Tris buffer, $\mathrm{pH} 7.4$ ). The sections were then blocked with $0.5 \% \mathrm{BSA}$ containing TS for 30 min and incubated with GFP chicken antibody in the blocking buffer overnight at room temperature. After washing, the sections were blocked in $0.8 \% \mathrm{BSA} / 0.1 \%$ gelatin-containing PB for 10 min and incubated with UltraSmall gold goat-anti-chicken antibody (Electron Microscopy Sciences) for $3 \mathrm{~h}$. The stained sections were postfixed with $2 \%$ glutaraldehyde for $10 \mathrm{~min}$ followed by silver enhancement for $4 \mathrm{~min}, \mathrm{OsO}_{4}$ fixation, and dehydration. The sections were then flat embedded in Epon and cut into 70-nm-thick ultrathin sections. The most superficial sections were counterstained and examined on a CM10 Electron Microscope (Philips).

C57BL/6J mice were transcardially perfused with $2.5 \%$ glutaraldehyde plus $4 \%$ PFA in $0.1 \mathrm{~m}$ sodium cacodylate buffer, $\mathrm{pH}$ 7.4. The prepared eyecups were stored in the same fixative solution at $4^{\circ} \mathrm{C}$ before being further processed. Small pieces of eyecup $(\sim 2 \times 2 \mathrm{~mm})$ were processed for en bloc fixation and staining by the following steps: (1) washes in cold cacodylate buffer containing $2 \mathrm{~mm}$ calcium chloride; (2) $1.5 \%$ potassium ferrocyanide, $2 \mathrm{~mm}$ calcium chloride, and $2 \%$ osmium tetraoxide in 0.15 M cacodylate buffer, pH 7.4 for $1 \mathrm{~h}$ on ice; (3) thiocarbohydrazide treatment for $20 \mathrm{~min}$ at room temperature; (4) $2 \%$ osmium tetroxide fixation 


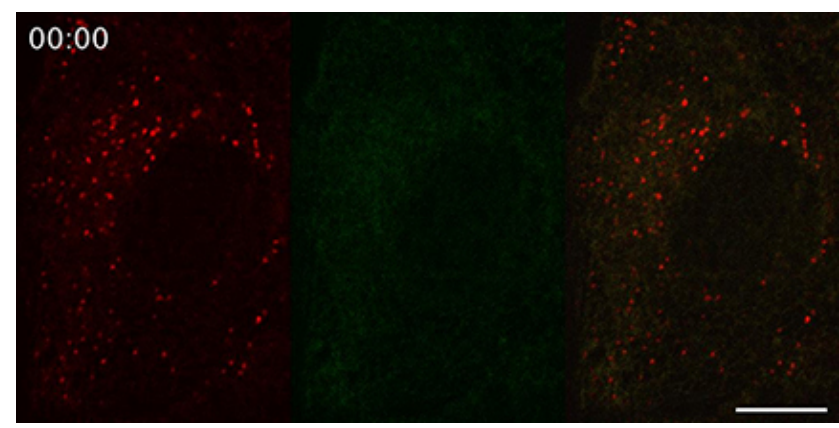

Movie 2. Time-lapse video recording of stably expressed WT PRPH2$\mathrm{mCh}-\mathrm{GFP}$. The majority of the granules exhibit red, but not green, fluorescence. Also visible are highly dynamic tubulovesicles, appearing to be shuttled between the red LE granules. Scale bar, $10 \mu \mathrm{m}$.
$\mathrm{N}$-ethylmaleimide, and spun again $\left(13,000 \mathrm{rpm}\right.$, for $15 \mathrm{~min}$, at $\left.4^{\circ} \mathrm{C}\right)$. The obtained postnuclear supernatants were subjected to immunoprecipitation using the antibody and Dynabeads Protein G (Thermo Fisher Scientific). The postnuclear supernatants of the transfected $293 \mathrm{~T}$ cell lysates were similarly prepared in RIPA buffer and subjected to immunoprecipitation assay. Phenylmethylsulfonyl fluoride and proteinase/phosphatase inhibitor cocktails were added during cell lysing. Quantitative immunoblotting assays were performed using the Odyssey Infrared Imaging System (LI-COR).

Experimental design and statistical analysis section. Power analysis was conducted to estimate the sample size using $G^{*}$ Power 3.1 software. All the statistical tests were performed by Student's $t$ test using a confidence interval of $95 \%$. All the graphs are presented as the mean \pm SD. We analyzed at least three mice for each transfection. To date, we have not encountered any difference in the transfection efficiency or morphology of retina to mouse sex, and thus both sexes were used. Details regarding the number of cells, particles, blots, and exact $p$ values are included in the figure legends.

\section{Results}

\section{PRPH2 transits through LE and recycling endosomes en route} to cilia

We first characterized the ciliary targeting of PRPH2 stably expressed in MDCK cells. Stable MDCK lines that expressed a similar modest level of various $\mathrm{PRPH} 2$ reporters were examined. The representative results obtained from two independent clones of each cell line are shown.

Bovine and human PRPH2 are 91\% identical overall, and their $\mathrm{C}$ termini are $85 \%$ identical. To rule out any species difference or tagging effect, we tested in parallel the nontagged bovine PRPH2, the C-terminal mCh-tagged bovine PRPH2 (PRPH2$\mathrm{mCh}$ ), and the N-terminal Flag-tagged human PRPH2. Regardless of the tagging or the type of the tagging, the WT PRPH2 was consistently present on the acetylated $\alpha$-tubulin (or detyrosinated tubulin)-labeled primary cilia extending from the apical surface of the polarized MDCK cells (Fig. $1 A-C$ ). While PRPH2 was absent from the gp135-labeled apical plasma membrane (Fig. $1 B)$, it was enriched in the Rab11a- and Rab11b-labeled recycling endosome immediately beneath the apical plasma membrane (Fig. 1C,D). Surprisingly, PRPH2 at that region was also closely associated with the Lamp2-labeled vacuoles (Fig. 1D).

Previous studies showed that shRNA-mediated Rab11 silencing can impede the polarization (and hence, likely the ciliogenesis) of MDCK cells (Bryant et al., 2010). To test whether cytoplasmic PRPH2 travels through the endosomes before entering the cilium, we allowed the PRPH2-GFP-expressing cells to polarize and form cilia first, and then induced the expression of dominant-negative mutant Rabs for $8 \mathrm{~h}$. Overexpressing mChRab7a T22N and mCh-Rab11a S25N (or mCh-Rab11b S25N) were used to selectively disrupt the trafficking of LE/Lys and recycling endosome, respectively. We performed FRAP assays and measured the recovered rate of the ciliary green fluorescence of PRPH2-GFP after photobleaching. The recovery of the ciliary PRPH2-GFP was detectably slower in Rab7a T22N-, Rab1la S25N-, or Rab11b S25N-expressing cells compared with the control cells (Fig. 1E,F). The results support the notion that the trafficking pathway of the LE/Lys and the recycling endosome are important for the ciliary entry of PRPH2.

Next, we tested a naturally occurring mutant PRPH2, 1137TG, which had the C-terminal 46 aa replaced by 89 different residues, due to the deletion of nucleotides 1137 and 1138 (Nichols et al., 1993). In contrast to the WT, the 1137TG mutant was largely absent from the primary cilium and subapical region, and, instead, was prominently aligned along the E-cadherin-labeled 
A

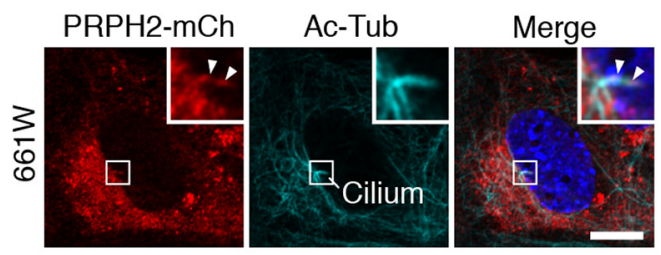

B

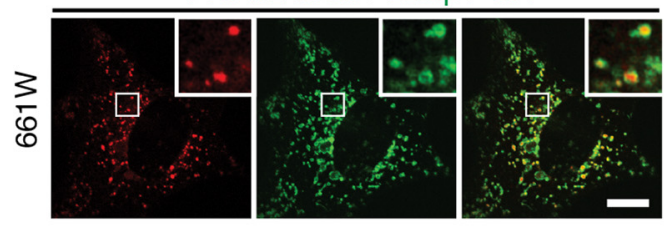

C

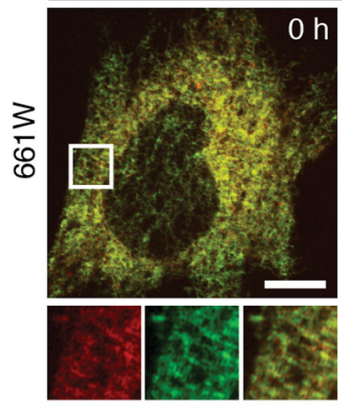

D

The red vacuole number per cell
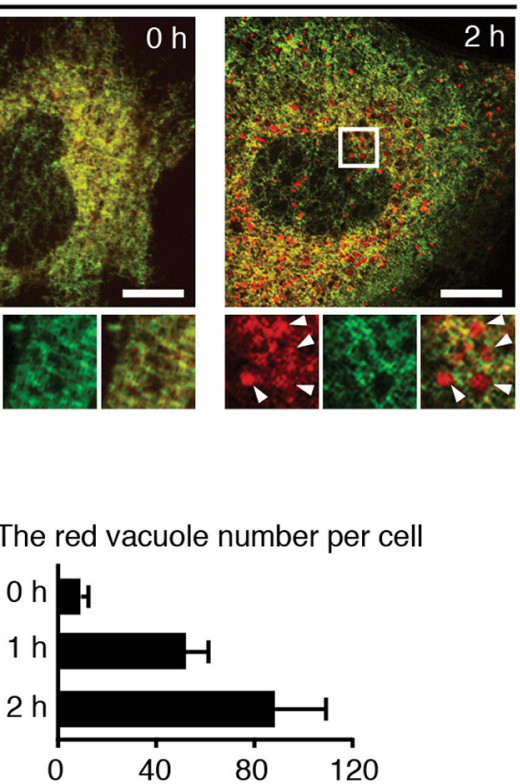
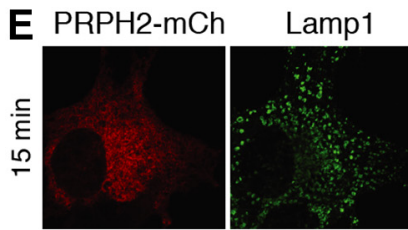

GM130
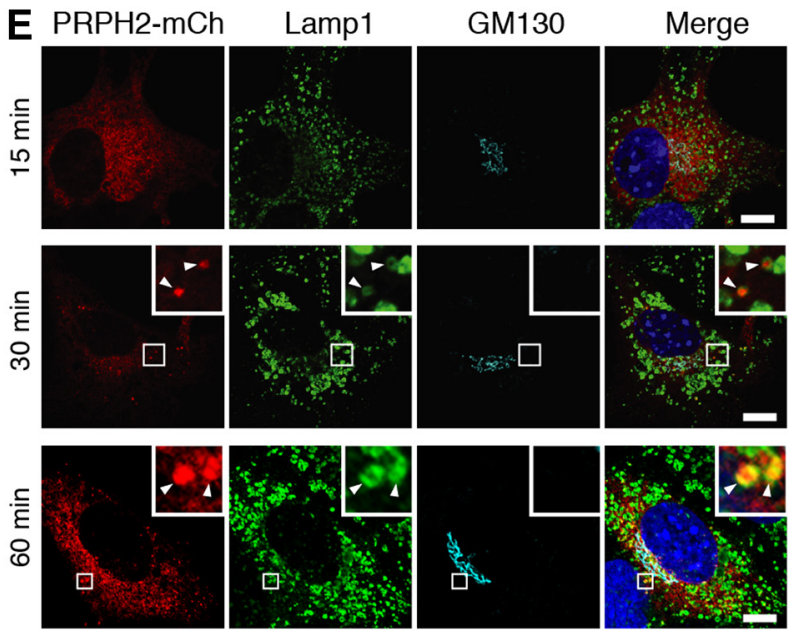

F $\mathrm{PRPH} 2-\mathrm{mCh}$

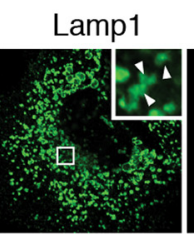

GM130

Merge
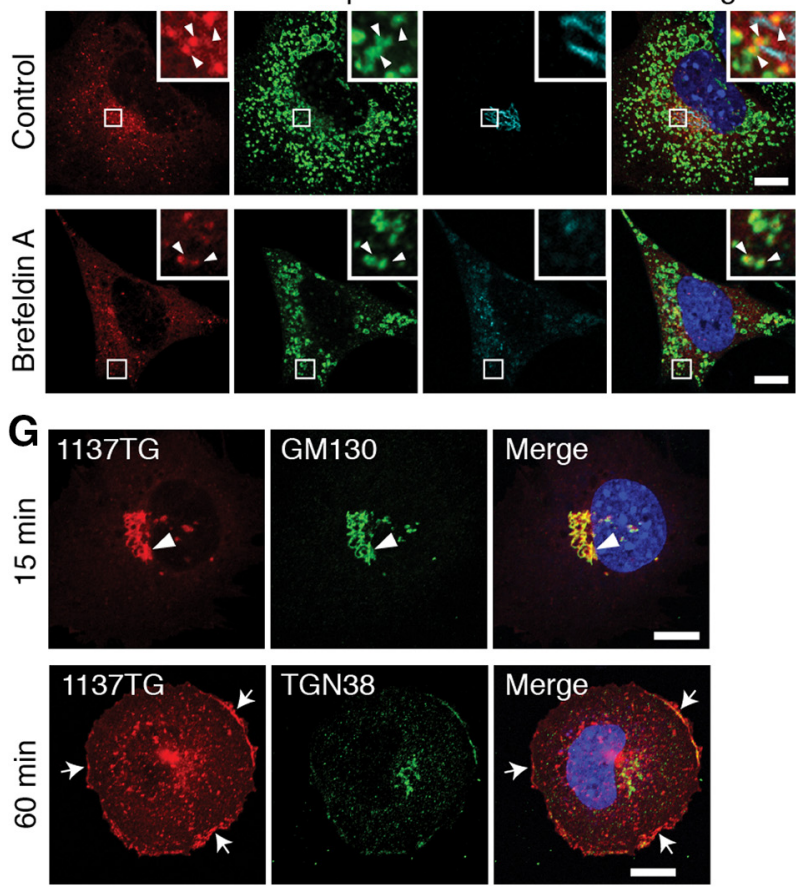

Figure 4. Newly synthesized PRPH2 undergoes the Golgi-independent ER-to-LE transport in $661 \mathrm{~W}$ cells. $A, 661 \mathrm{~W}$ cells stably expressing PRPH2-mCh were serum starved to induce cilium formation and then processed for staining of $\mathrm{mCh}$ and acetylated $\alpha$-tubulin (Ac-Tub). Insets (enlarged boxed areas) highlight the cilium localized PRPH2-mCh (arrowheads). $\boldsymbol{B}$, Representative still images, in both low-power and high-power (insets) magnifications, show that transfected PRPH2-mCh and Lamp1-GFP are closely associated in live 661W cells. C, D, PRPH2-mCh-GFP transfected in $661 \mathrm{~W}$ cells for $4 \mathrm{~h}$ was synchronized at the $\operatorname{ER}\left(15^{\circ} \mathrm{C}, 2 \mathrm{~h}\right)$ and chased for the indicated durations. Representative confocal images $(\boldsymbol{C})$ and quantification of the number of red-only vacuoles $(\boldsymbol{D}$; mean $\pm S D ; n=30$ cells, $N=3$ ) are shown. $E$, PRPH2-mCh transiently expressed in $661 \mathrm{~W}$ cells for $4 \mathrm{~h}$ were synchronized in the $E R\left(15^{\circ} \mathrm{C}, 2 \mathrm{~h}\right)$ and chased for the indicated times. Representative confocal images of $\mathrm{mCh}$, Lamp1, and GM130 immunostaining are shown. Arrowheads mark the overlapping signal of Lamp1 with ER-released PRPH2-mCh. $\boldsymbol{F}, \mathbf{G}$, WT $(\boldsymbol{F})$ or $1137 \mathrm{TG}(\boldsymbol{G})$ PRPH2-mCh expressed in $661 \mathrm{~W}$ cells for $4 \mathrm{~h}$ was blocked at the ER and then released in the absence (control in $\boldsymbol{F} ; \boldsymbol{G}$ ) or the presence of brefeldin $\mathrm{A}$ for $2 \mathrm{~h}(\boldsymbol{F})$ or for the indicated duration $(\boldsymbol{G})$. The cells were then stained with the indicated antibodies. Arrowheads in $F$ show that the nascent WT PRPH2 was on LE but not on Golgi. Arrow and arrowheads in $\boldsymbol{G}$ point to the cis-Golgi and cell border signal of $1137 T G$, respectively. Blue, DAPI nuclei $(\boldsymbol{A}, \boldsymbol{E}, \boldsymbol{F}, \boldsymbol{G})$. Scale bars: $\boldsymbol{A}-\boldsymbol{C}, \boldsymbol{E}-\mathbf{G}, 10 \mu \mathrm{m}$.

lateral membranes (Fig. $1 A, B$ ). These results indicate that the $\mathrm{C}$ terminus of PRPH2 contains the sorting signal for its apical ciliary expression.

\section{WT PRPH2, but not 1137TG mutant, is expressed in the} lumen of nondegradative $\mathrm{LE}$

We then performed a series of colocalization studies to comprehensively document the subcellular distribution of PRPH2. We conducted these experiments in subconfluent cells for better spatial resolution. Since several organelle markers and the antibovine PRPH2 antibody were both made in mice, we first cola- beled the GFP-tagged organelle-specific reporters with the stably expressed nontagged bovine PRPH2 (Fig. 2A). The Pearson's coefficients showed that, among all the organelles tested, PRPH2 had the highest association with Lamp1-GFP-labeled (Fig. 2A) and GFP-CD63-labeled (data not shown) LE/Lys (Fig. 2B). A smaller subset of PRPH2 was expressed on GFP-Rab11a-labeled recycling endosomes and GFP-EEA1-labeled early endosomes (Fig. 2A,B). Galactosyltransferase-labeled TGN and plasma membrane had no detectable PRPH2 (Fig. 2A, $B$ ).

The predominant LE/Lys expression of PRPH2 was also confirmed by the colabeling of the stably expressed PRPH2-mCh 


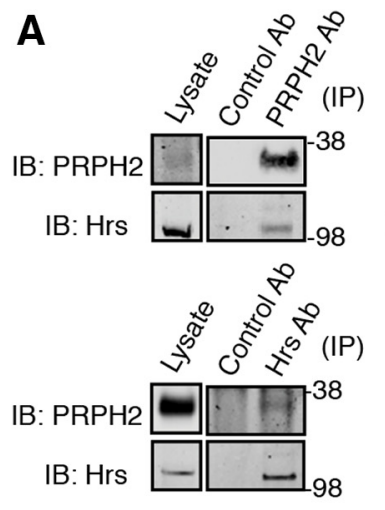

D

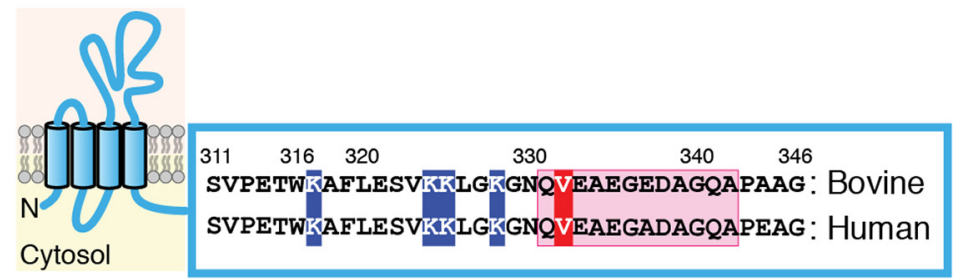

B

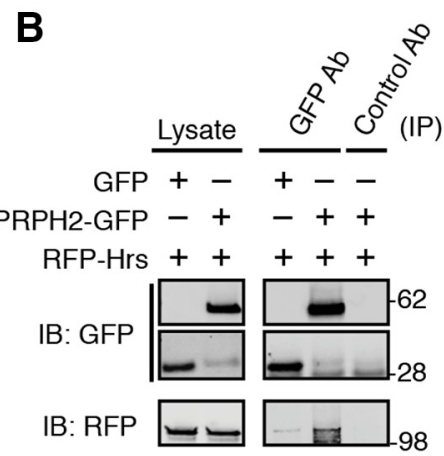

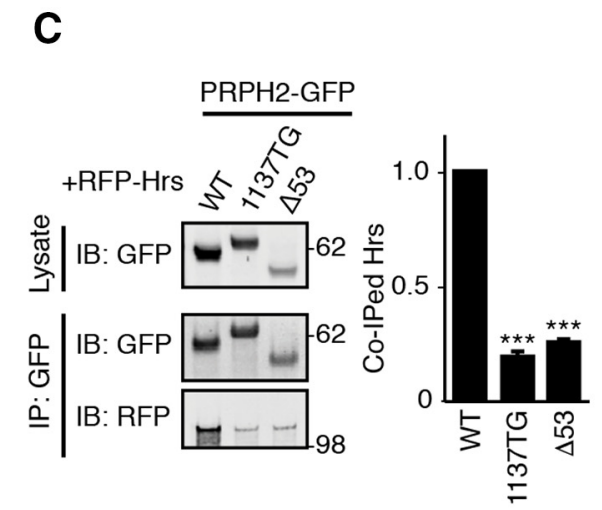

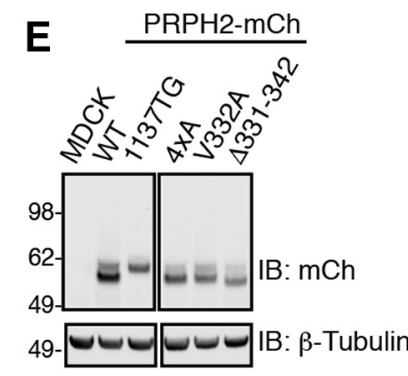

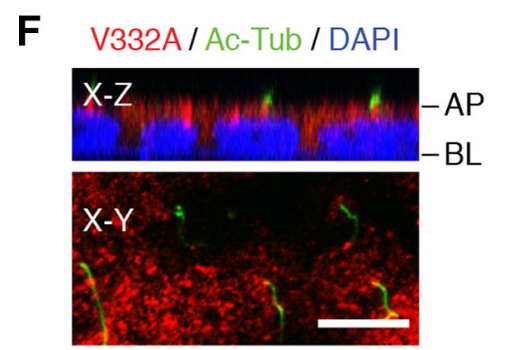
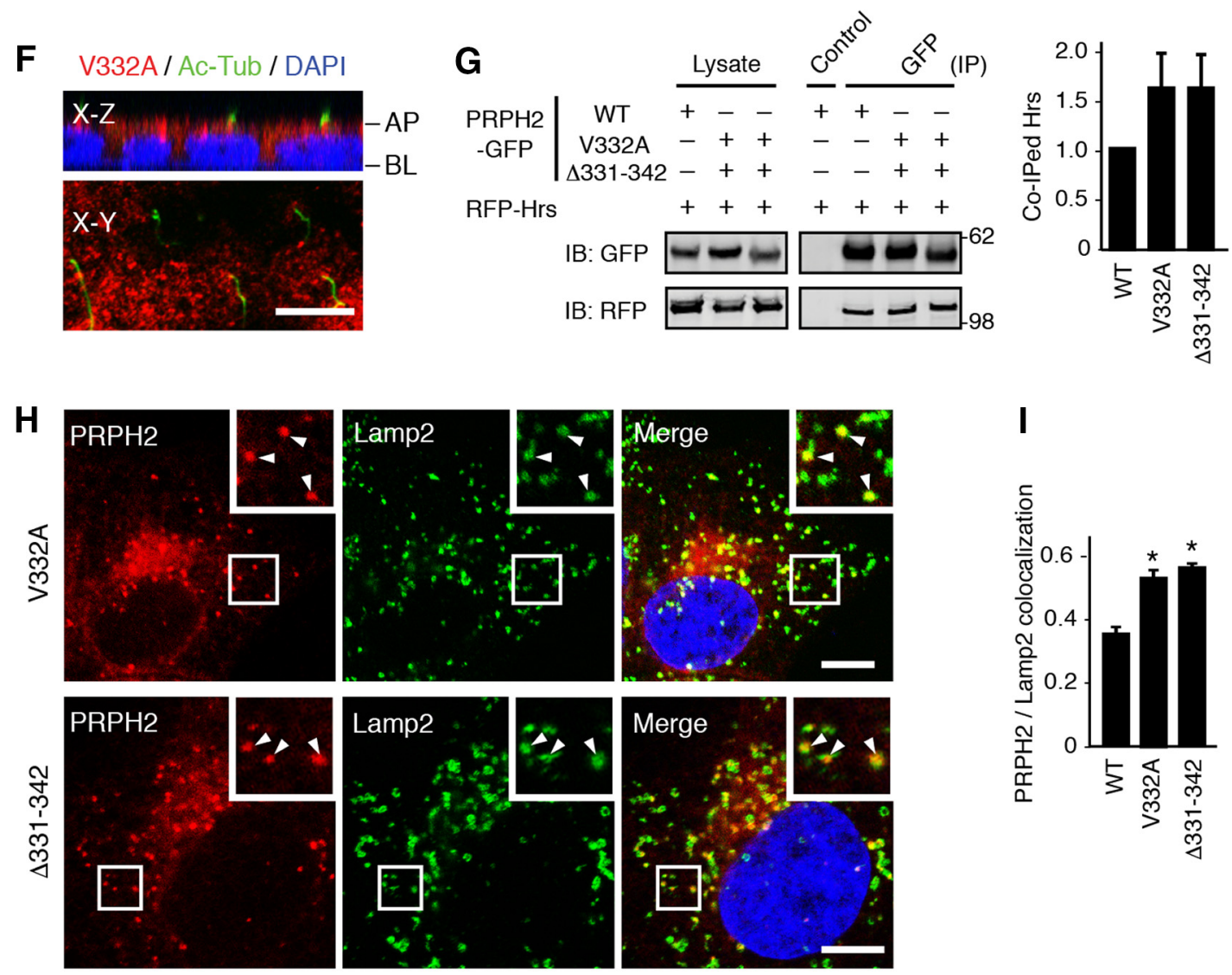

Figure 5. Delineate the C-terminal motifs of PRPH2 that distinctively regulate its LE and ciliary targeting. $A$, Total detergent lysates of mouse retinas and immunoprecipitates of PRPH2 (top) or Hrs (bottom) obtained from retinal lysates were immunoblotted with the indicated antibodies. $\boldsymbol{B}$, Total lysates of 293T cells transfected with RFP-Hrs and PRPH2-GFP or GFP, and the respective immunoprecipitates pulled down by control or GFP antibody were immunoblotted as indicated. C, Total lysates of 293T cells cotransfected with RFP-Hrs with the indicated PRPH2-GFP variants, as well as their respective GFP immunoprecipitates were immunoblotted as indicated. Considering the amount of Hrs pulled down by WT PRPH2 as 1, the relative amounts of Hrs pulled down by the indicated PRPH2 variants are shown. 1137TG, $p=0.00024 ; \Delta 53, p=0.00023$. D, Schematic diagram of the membrane topology of PRPH2 and the cytoplasmic C-terminal amino acids of WT bovine and human PRPH2 in which the valine 332, the region encompassing residues 331-342, and the four conserved lysines (K) are highlighted in red, pink, and blue, respectively. $E$, Immunoblots of MDCK lines stably expressing the PRPH2-mCh variants characterized in this article using the indicated antibodies. All PRPH2 variants have the expected molecular masses; they did not exhibit any detectable aggregation or degradation. $\boldsymbol{F}, x-z$ and $x-y$ confocal images of MDCK monolayers stably expressing the V332A mutant of PRPH2-mCh and stained for mCh, acetylated $\alpha$-tubulin (Ac-Tub), and DAPI. AP, Apical side; BL, basolateral side. G, Representative images of immunoprecipitation assays of Hrs coimmunoprecipitated by the WT vs the indicated (Figure legend continues.) 
with the endogenous Lamp2 (Fig. 2C) and Lamp1-GFP (Fig. 2D, Movie 1). Furthermore, in agreement with the notion that the LE/Lys is tethered to the ER network (Eden et al., 2012; Friedman et al., 2013), PRPH2-labeled granules were affiliated with GFPSec61 $\beta$-labeled ER tubules (Fig. 2E). Little or no PRPH2-mCh was detected on the GM130-labeled cis-Golgi (Fig. 2D).

We performed a fluid-phase tracer experiment to independently demonstrate the LE/Lys expression of PRPH2. As shown in Figure $3 A$, the extracellularly fed green fluorescein-conjugated dextran, which is known to accumulate in the LE lumen, had extensive overlap with stably expressed PRPH2-mCh.

The late endocytic compartment is known to be heterogeneous; not all of the LEs merge to become lysosomes (Kobayashi et al., 2002; White et al., 2006; Piper and Katzmann, 2007; Edgar et al., 2014). We used the extracellularly internalized EGF to selectively mark the population of LE that fuses with the Lys (Felder et al., 1990). As described previously (Kajimoto et al., 2013), to visualize the luminal content in slightly swollen LE/Lys, we analyzed the internalized Alexa Fluor 647-EGF in the PRPH2expressing MDCK cells transfected with GFP-CD63 and Rab5Q79L (Fig. 3B). These experiments showed that the majority of PRPH2 was expressed in the Alexa Fluor 647 EGF-negative, CD63-positive granules (Fig. 3 C). Thus, PRPH2 is mainly expressed in the nondegradative LE, rather than the degradative LE/Lys.

In the LEs, PRPH2 was surrounded by the limiting membrane highlighted by Lamp1, Lamp2, or CD63 (Figs. 1D, 2A, 3B). This was especially evident when super-resolution confocal microscopy was used (Fig. $1 D$, right). Consistently, PRPH2 extensively overlapped with the dextran-filled LE (Fig. $3 A$ ). To formally demonstrate that PRPH2 is localized to the luminal side of the LE, we assessed the $\mathrm{pH}$ of the milieu encountered by the PRPH2 C terminus, which was tagged by both $\mathrm{mCh}$ and GFP in tandem (PRPH2-mCh-GFP). The GFP-derived green fluorescence, but not the mCh-derived red fluorescence, is susceptible to quenching by low $\mathrm{pH}$. The superfolder version of GFP was used in this construct and the constructs herein prevent molecular aggregation (Pédelacq et al., 2006). In both fixed and live cells, most of the PRPH2-mCh-GFP puncta expressed only red fluorescence (Fig. 3D, top panels; Movie 2). Raising the luminal $\mathrm{pH}$ by treating the cells with $\mathrm{NH}_{4} \mathrm{Cl}$ effectively recovered the green fluorescence, which matched the location of the red fluorescence and merged to form a yellow signal (Fig. 3D, middle panels).

Compared with the WT, 1137TG had significantly reduced Lamp2 colocalization (Fig. $3 E, F$ ), indicating that the mutant had impaired LE targeting. Further supporting this, in contrast to its WT counterpart, the 1137TG mCh-GFP reporter exhibited both red and green fluorescence (Fig. 3D, bottom panels). 1137T was prominently detected on the cell border (Fig. $3 D, F, G$, arrows) and, to a less extent, on the early endosome in subconfluent MDCK cells (Fig. $3 G$ ).

\footnotetext{
$\leftarrow$

(Figure legend continued.) mutant PRPH2. The relative amount of coimmunoprecipitated Hrs is shown at the right (not significant). $\boldsymbol{H}$, Representative confocal images of MDCK cells stably expressing the indicated PRPH2-mCh variants. The cells were labeled with Lamp2. Arrowheads point to the colocalized signals. $\boldsymbol{I}$, Quantification of $\boldsymbol{H}$. Pearson's coefficients representing the colocalization between Lamp2 and the indicated PRPH2 variants (mean \pm SD; $n=24$ cells, $N=3$ ). V332A, $p=0.019 ; \Delta 331-342, p=0.0064 .{ }^{* * *} p<0.0005 ;{ }^{*} p<0.05$, Student's $t$ test. Scale bars: $\boldsymbol{F}, \boldsymbol{H}, 10 \mu \mathrm{m}$.
}

\section{Golgi-independent ER-to-LE transport of PRPH2 requires its C terminus}

To further delineate the biosynthetic pathway of $\mathrm{PRPH} 2$, we used a more relevant cell model, a cone cell line $661 \mathrm{~W}$. We found that serum-starved quiescent $661 \mathrm{~W}$ cells grew primary cilia. Since endogenous PRPH2 was undetectable in these cells (Tan et al., 2004), we showed that the stably expressed PRPH2-mCh was present on the cilia of the 661W cells (Fig. 4A). Furthermore, PRPH2-mCh was also expressed in the Lamp1-GFP-labeled LEs (Fig. 4B). The extent of PRPH2-Lamp1 colocalization was significant and was similar in the cells cultured with (Pearson's coefficient $=0.31 \pm 0.08 ; 24$ cells; $N=3$ ) or without (Pearson's coefficient $=0.31 \pm 0.09 ; 24$ cells; $N=3$ ) serum.

The absence of the PRPH2 signal from the plasma membrane and its expression in the LE prompted us to ask whether the LE is in the biosynthetic pathway of nascent PRPH2. To test this, we subjected the newly synthesized ( $4 \mathrm{~h}$ post-transfection) PRPH2mCh-GFP in $661 \mathrm{~W}$ cells to $15^{\circ} \mathrm{C}$ ER block and then $37^{\circ} \mathrm{C}$ chase. At the $0 \mathrm{~h}$ time point (i.e., before chase), the ER-held PRPH2mCh-GFP had both red and green fluorescence (Fig. 4C). Little or no red granular signal was observed (Fig. 4D). The number of "red-only" vacuoles (likely the acidic LEs) gradually increased over the $2 \mathrm{~h}$ period of chase (Fig. $4 C, D)$. At no time point $(15 \mathrm{~min}$, $30 \mathrm{~min}, 1 \mathrm{~h}, 2 \mathrm{~h}$ ) was PRPH2 found on the cell surface or the Golgi cell (Fig. 4E). Importantly, PRPH2 was still chased into the red-labeled granules in the cells treated with brefeldin A, a Golgidisrupting agent (Fig. 4F). In stark contrast to its WT counterpart, the 1137TG-mCh was mainly chased into the Golgi cell (15 min), and then the cell border (60 min; Fig. 4G).

These results suggest that the majority of the ER-released WT PRPH2 is delivered to the LE without first passing through the Golgi or the plasma membrane. Additionally, the C-terminal tail of PRPH2 contains a sorting signal for its ER-to-LE pathway. The PRPH2 mutant with this "dominant" signal removed undergoes the canonical Golgi-secretory pathway.

\section{The $\mathrm{C}$ terminus of PRPH2 is ubiquitinated, binds to Hrs, and contains multiple endosomal and ciliary targeting motifs}

Next, we investigated the mechanism underlying the LE targeting of PRPH2. Hrs has an established role in sorting various membrane cargoes into LE lumens (Raiborg and Stenmark, 2002). We showed that PRPH2 and Hrs physically interacted. In immunoprecipitation experiments from mouse retinal lysates with either the anti-PRPH2 antibody or the anti-Hrs antibody, the endogenous PRPH2 and Hrs were specifically coimmunoprecipitated with each other (Fig. 5A). Furthermore, transfected PRPH2-GFP, but not GFP alone, also pulled down the cotransfected RFP-Hrs in 293T cells (Fig. 5B). Significantly less RFP-Hrs was pulled down by the 1137TG-GFP compared with the WT-GFP (Fig. 5C). The GFP fusion of $\Delta 53$ mutant PRPH2 (missing the terminal 53 residues) also had decreased binding to the cotransfected RFP-Hrs (Fig. 5C). These results suggest that Hrs binds to the $\mathrm{C}$-terminal region of PRPH2.

Using chimera or deletion mutants in transgenic frog rods (Salinas et al., 2013) or transfected RPE-1 cells (Tian et al., 2014), previous studies showed that amino acids 331-342 and valine 332 at the distal C-terminal region of PRPH2 (Fig. 5D) are required for its ciliary expression through an unknown mechanism. We generated and characterized the MDCK lines expressing the $\mathrm{mCh}$ fusion of $\Delta 331-342$ (with the 331-342 residues deleted) and V332A (valine 332 was changed to alanine) mutant PRPH2 (Fig. $5 E$ ). Both the $\Delta 331-342$ mutant (data not shown) and the V332A mutant (Fig. $5 F$ ) failed to be expressed in the cilia of the polarized 
A

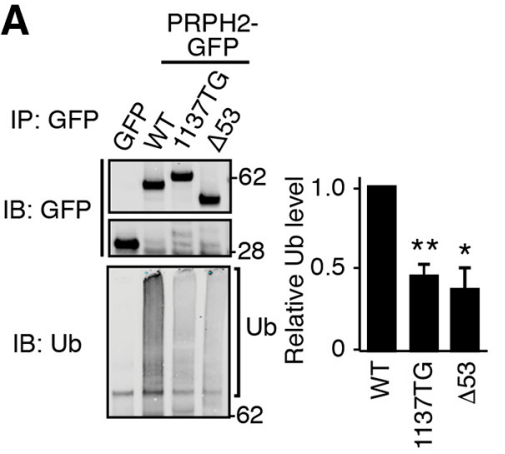

B

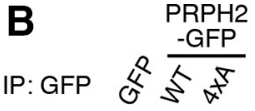

IB: GFP
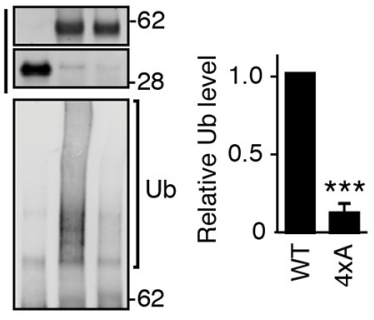

C

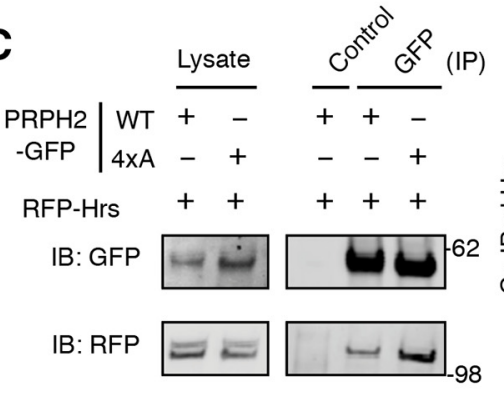

E
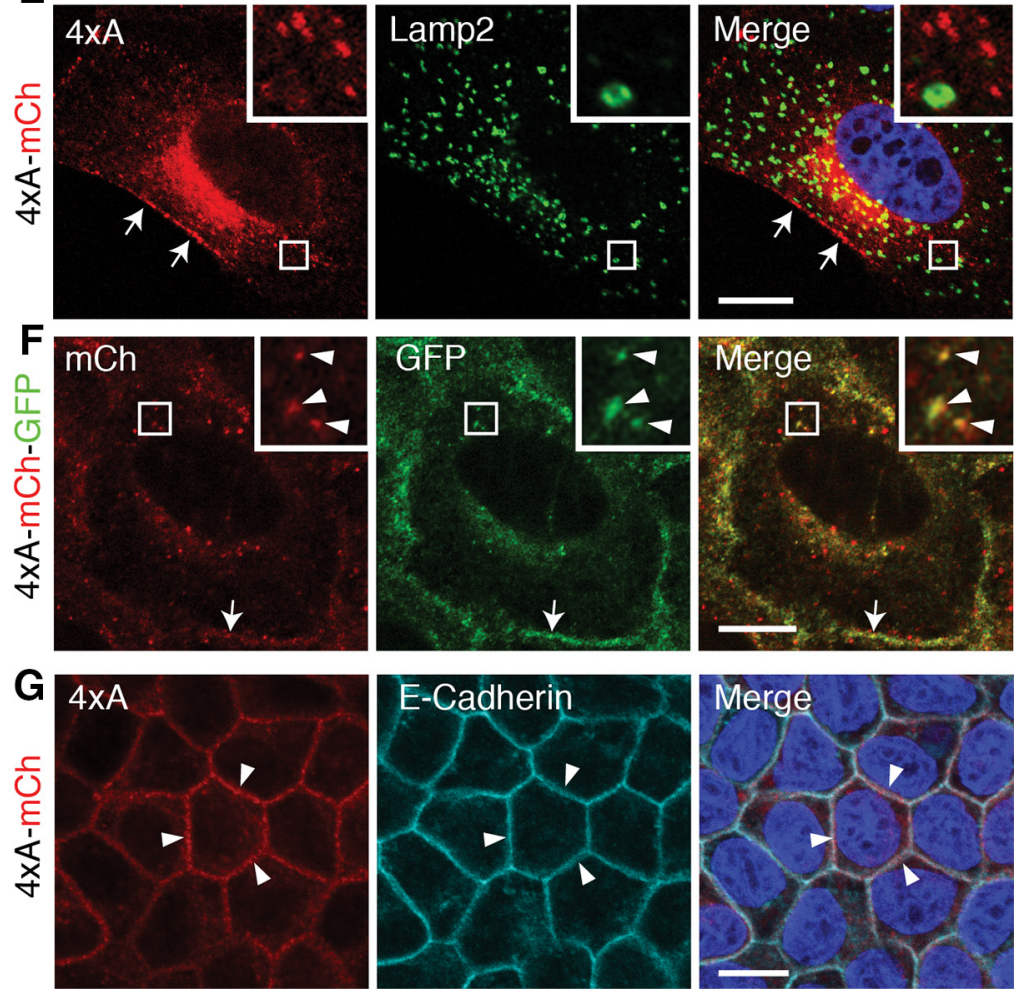

Figure 6. The C-terminal lysins of PRPH2 are ubiquitinated and involved in the LE and ciliary targeting. $\boldsymbol{A}, \boldsymbol{B}, \mathrm{GFP}$ immunoprecipitates from 293T cell lysates containing transfected GFP or PRPH2-GFP variants were immunoblotted for GFP or ubiquitin (Ub). Considering the ubiquitination of WT PRPH2 as 1, the relative ubiquitination level for the indicated PRPH2 variants are shown. 1137TG, $p=0.0091 ; \Delta 53, p=0.018(\boldsymbol{A}) .4 \times \mathrm{A}: p=0.00013(\boldsymbol{B})$. C, Representative immunoblots supporting the quantification demonstrate that RFP-Hrs was specifically pulled down by both WT and 4xA PRPH2-GFP. The relative amount of Hrs coimmunoprecipitated by the WT vs $4 \times A$ PRPH2 are shown at the right (not significant). $D$, Pearson's coefficients representing the colocalization between Lamp2 and 4xA PRPH2 (mean $\pm S D ; n=24$ cells, $N=3$ ). $p=0.0062$. $E$, Representative confocal images supporting the Lamp2 colocalization quantification in $\boldsymbol{D}$. MDCK cells stably expressed 4xA PRPH2-mCh, and PRPH2 was found in the cell borders (arrows) but rarely in the Lamp2-positive LE. $F$, Direct fluorescence of MDCK stably expressed 4xA PRPH2-mCh-GFP in intracellular granules (arrowheads) and cell border (arrows). G, The $x-y$ sectioning plane confocal images of $m C h$ and E-cadherin staining show that stably expressed 4xA PRPH2-mCh was prominently expressed on cell borders (arrowheads). ${ }^{* * *} p<0.0005$; ${ }^{* *} p<0.005 ;{ }^{*} p<0.05$ (Student's $t$ test). Scale bar: $\boldsymbol{E}-\mathbf{G}, 10 \mu \mathrm{m}$.
MDCK cells. Interestingly, both mutants still bound to Hrs (Fig. $5 G$ ), and, in fact, both mutants had statistically significant increased association with Lamp2-labeled LE (Fig. $5 H, I$ ). These results indicate that while these two distal C-terminal mutants can enter the LE, they fail to leave the LE for the subsequent ciliary targeting.

Ubiquitins added to the cytoplasmic region of several membrane proteins have been shown to be the LE lumen sorting signal that binds to Hrs (Raiborg and Stenmark, 2002). We found that WT PRPH2-GFP, but not GFP alone, expressed in transfected 293T cells was ubiquitinated (Fig. 6A). Both 1137TG and $\Delta 53$ had significantly reduced ubiquitination (Fig. 6A), supporting the idea that the $\mathrm{C}$ terminus is the major ubiquitination site for PRPH2. Subsequently, we generated the $4 \mathrm{xA}$ mutant, in which all four conserved lysines (K317, K324, K325, and K328; Fig. $5 D$ ) in the cytoplasmic tail of human PRPH2 were replaced by alanines. The 4xA mutation underwent significantly less ubiquitination (Fig. 6B), indicating that the $\mathrm{C}$-terminal lysine residues are important ubiquitination sites. Unexpectedly, while the $4 \mathrm{xA}$ mutant still bound to Hrs (Fig. 6C), it had defective LE targeting. The $4 x A-m C h$ had reduced Lamp2 colocalization (Fig. $6 D, E$ ), and the $4 \mathrm{xA}$ mCh-GFP reporter displayed both red and green fluorescence (Fig. $6 F$ ). Furthermore, $4 \mathrm{xA}$ was not detected in the primary cilium (data not shown). The missorted $4 \mathrm{xA}$ was readily detected in the cell borders (Fig. $6 E-G$ ). These results suggest that the four lysines in the $\mathrm{C}$ terminus of PRPH2 are important for the LE and ciliary targeting of $\mathrm{PRPH} 2$ through an Hrsindependent pathway.

\section{Characterizing the expression of LE and PRPH2 in photoreceptor}

Each photoreceptor has the following four major compartments: the outer segment, the inner segment, the cell body/cell process, and the synapse (Fig. 7A). Outer segment proteins are synthesized by the biosynthetic pathways housed in the cell body and the inner segment. The endosomes of mammalian photoreceptors remain poorly characterized. Using transmission EM, the LEs, characterized by their multivesicular vacuoles (Fig. $7 B$, top left), were readily seen in the photoreceptor inner segments and cell bodies. Typical looking lysosomes containing an electron-dense luminal matrix were, however, rarely seen.

To further delineate the distribution pattern of the LEs in the photoreceptors 

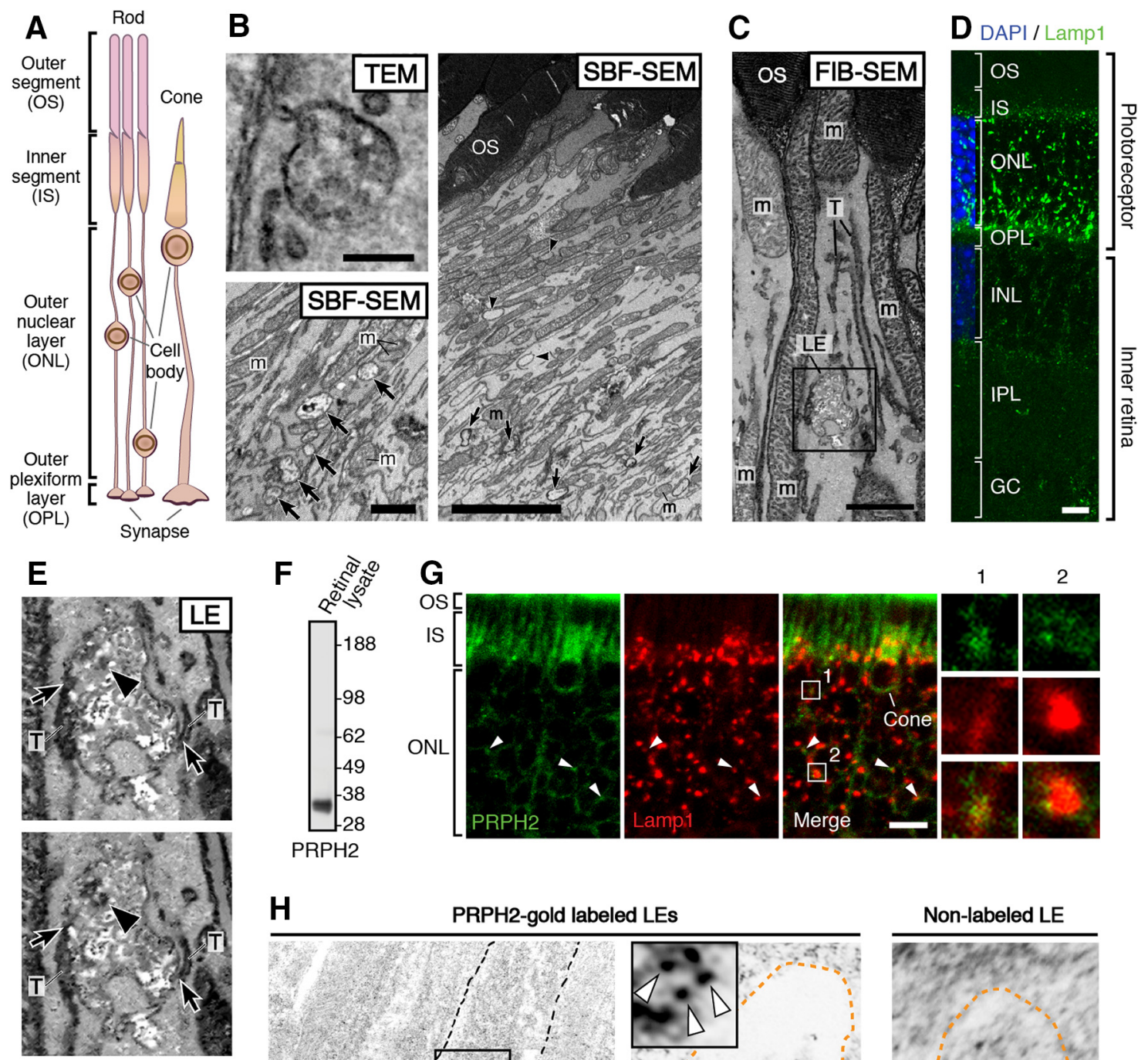

$\mathrm{PRPH} 2$
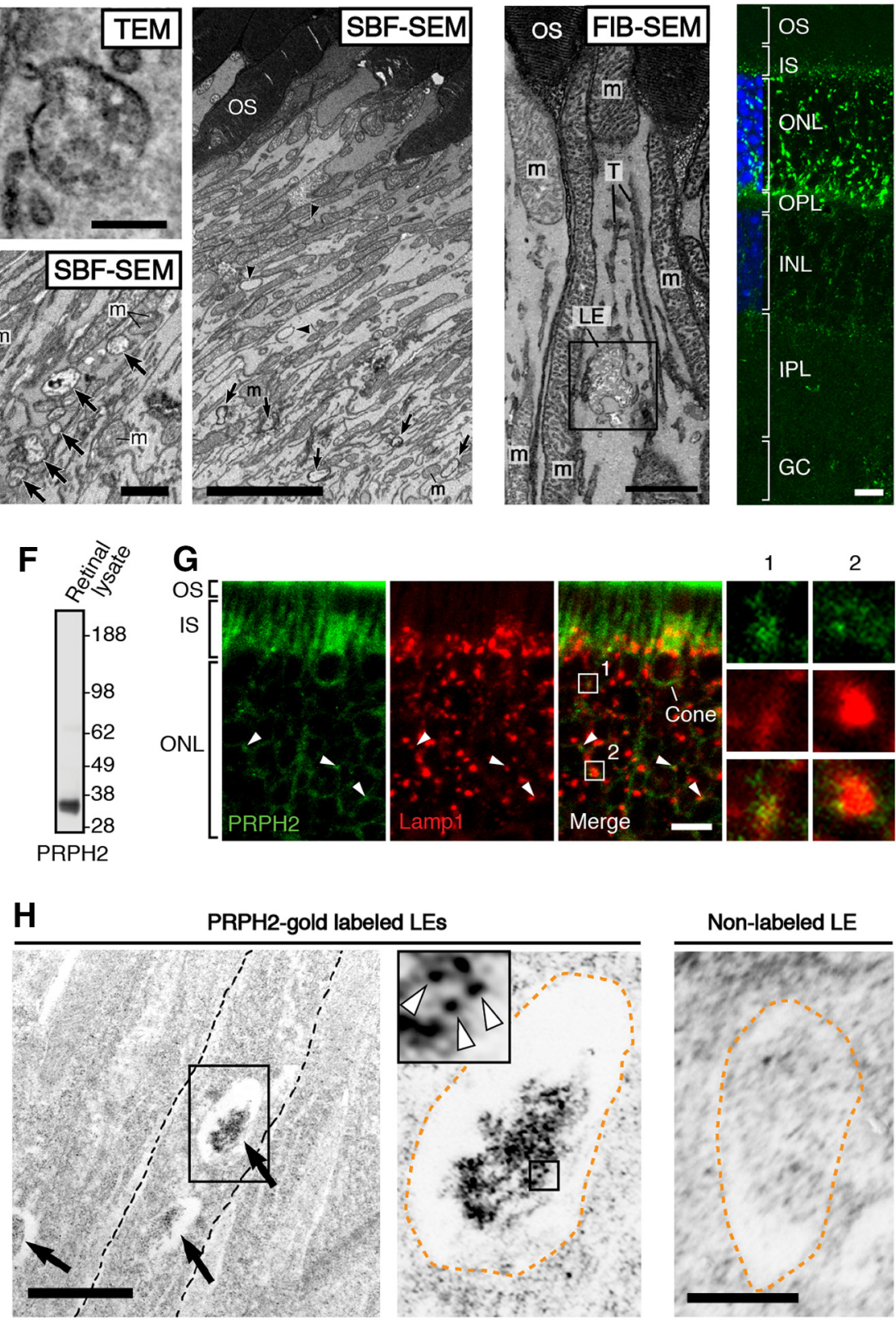

Figure 7. Characterization of LE and LE expression of PRPH2 in rodent photoreceptors. $\boldsymbol{A}$, A schematic drawing of mammalian photoreceptors, both rod and cone. $\boldsymbol{B}$, Top left, A representative multivesicular vacuole, characteristics for LE, seen in the mouse inner segment by transmission EM (TEM). Right, A low-power, single-view plane of a SBF-SEM shows the photoreceptor region containing the basal outer segments (OSs) and part of the inner segments of an adult CD1 mouse retina. Arrowheads point to the typical LEs; their multivesicular feature was confirmed by surveying the entire stack of zoomed-in images. Bottom left, An SBF-SEM image of a cone inner segment (IS) that contains abundant LEs (arrows). This cone was identified by its typical cone outer segment when a stack of images was viewed in high power. Mitochondria (m). C, A single FIB-SEM image shows LE-like vacuole (boxed), dark membrane tubules $(\mathrm{T})$, and mitochondria $(\mathrm{m})$ in a mouse photoreceptor inner segment. $\boldsymbol{D}$, A representative confocal image of the entire mouse retina immunostained with Lamp1. The DAPI dye labels the cell bodies of photoreceptors [i.e., the outer nuclear layer (ONL)] and the cell bodies of the inner retinal cells [i.e., the inner nuclear layer (INL)]. Detectably less Lamp1 signal was seen in the nonphotoreceptor layers. OPL, Outer plexiform layer; IPL, inner plexiform layer; GC, ganglion cell layer. $E$, Three FIB-SEM views (in $25 \mathrm{~nm}$ intervals) of the same LE boxed in C (Movie 3), which has dark luminal membranes (arrowheads) and is connected to dark tubules ( $T$; arrows) distributed alongside the long axis of the inner segments. The quantification of eight FIB-SEM electron micrographs of two representative LEs (shown in Movies 3,4 ) suggests $39.0 \pm 12.1 \%$ of the total luminal membranes are darkly stained. $\boldsymbol{F}$, The immunospecificity of the anti-PRPH2 rabbit antibody was confirmed by immunoblotting assay using mouse retinal lysates. G, Representative PRPH2 and Lamp1 staining in photoreceptors of human retinas. Arrowheads mark some overlapping PRPH2 (green) and Lamp1 (red) signal spanning the ONL. To emphasize the PRPH2 signal in the rest of photoreceptor, only the basal portion of the outer segment (OS) is shown. Enlarged views of box 1 and box 2 highlight the fine PRPH2 punctates associated with Lamp1-labeled granules. $\boldsymbol{H}$, ImmunoEM of rat photoreceptors expressing PRPH2-GFP for $16 \mathrm{~h}$. Left, A low-power electron micrograph shows three immunogold-decorated LEs (arrows). Dashed lines demarcate the borders between individual inner segments. Middle, An enlarged view shows an LE having a high-density immunogold-labeled lumen. Orange dashed lines mark the border of the LE vacuole. White arrowheads in the enlarged view of the boxed area (inset) point to the PRPH2-derived silver-enhanced immunogold labeling. Right, A representative image of LE lacking immunogold labeling. Scale bars: left top in $\boldsymbol{B}, 200 \mathrm{~nm}$; left bottom in $\boldsymbol{B}, \boldsymbol{C}, \boldsymbol{D}, 1 \mu \mathrm{m}$; right in $\boldsymbol{B}, 4 \mu \mathrm{m} ; \boldsymbol{D}, \boldsymbol{G}, 20 \mu \mathrm{m}$; left in $\boldsymbol{H}, 500 \mathrm{~nm}$; right in $\boldsymbol{H}, 250 \mathrm{~nm}$. 


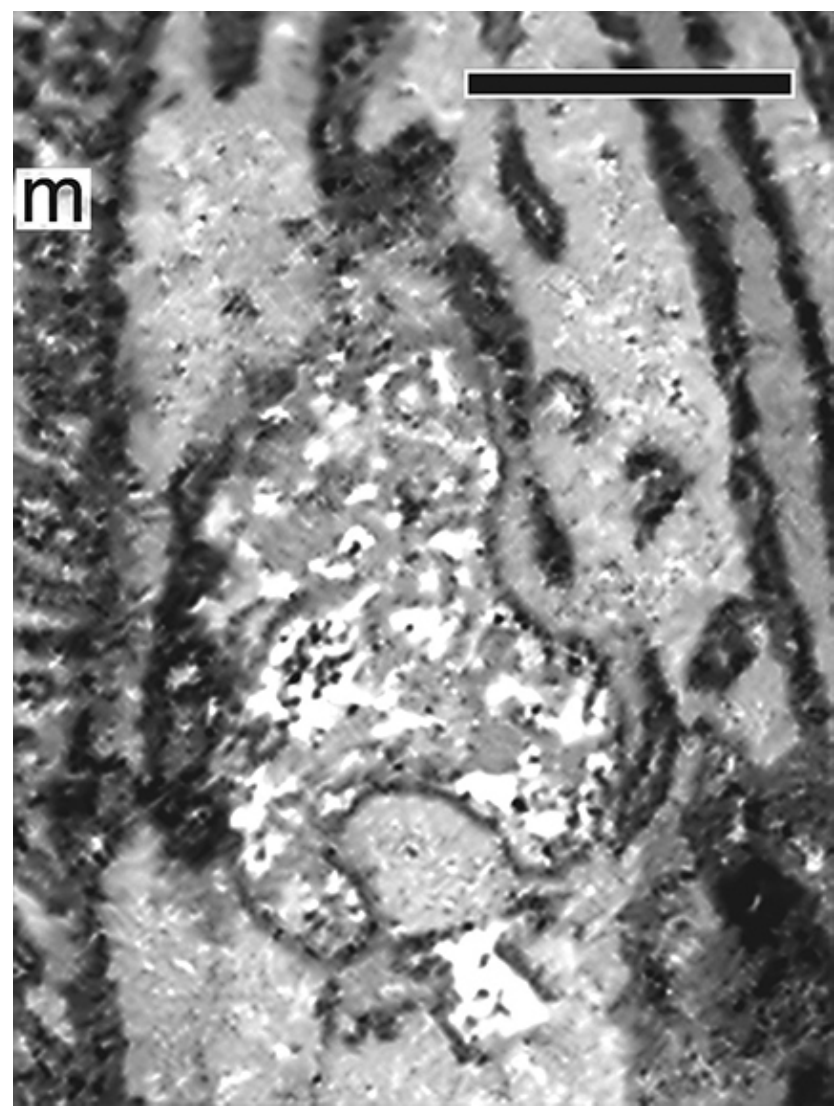

Movie 3. An FIB-SEM movie presents a series of consecutive block face images of a single LE in the mouse photoreceptor inner segment (i.e., " $\mathrm{LE}$ " shown in Fig. $7 E ; 5 \mathrm{~nm}$ per frame; total of 20 frames). These images show that the LE is highly polymorphic, and it has close contacts with the dark membrane tubules (T) belonging to a tubular network that runs longitudinally across inner segment. An arrowhead indicates the high-electron density intraluminal vesicle contents. m, mitochondria. Scale bar, $500 \mathrm{~nm}$.

and the geometry of individual LEs, we used two types of 3D EM techniques. Namely, we performed serial block-face viewing of "en block-fixed" (vs ultrathin-sectioned) retinal tissues, by either serial block-face scanning EM (SBF-SEM; Fig. 7B) or FIB-SEM (Fig. 7C). We found that the LE vacuoles were more frequently seen in the lower part of the inner segment of both rods and cones (Fig. $7 B$ ). This was consistent with the Lamp1 immunostaining of mouse retinal sections (Fig. 7D). Although bright Lamp 1 puncta were also found in the cell body (i.e., the outer nuclear layer) and the terminus (i.e., the outer plexiform layer) of the photoreceptors, we did not perform detailed EM imaging of those regions.

The high-power 3D topographies revealed that many of the LEs were pleomorphic. Their lumens were filled with vesicular tubules of variable electron density (Fig. 7E; Movies 3, 4, 5). A variable subset of these luminal membrane had matched electron density to the membrane tubules that were attached to and/or extended from the vacuoles, which appeared to belong to a tubular network spanning nearly the entire inner segment. The dark tubules $(\sim 35-75 \mathrm{~nm}$ in diameter) morphologically resembled endosomal tubules described in the literature (Tooze et al., 1990; Gibson et al., 1998).

The immunostaining of human retinal sections showed that, in addition to the bright outer segment signal, a modest, but specific, fine punctate signal of PRPH2 was also associated with

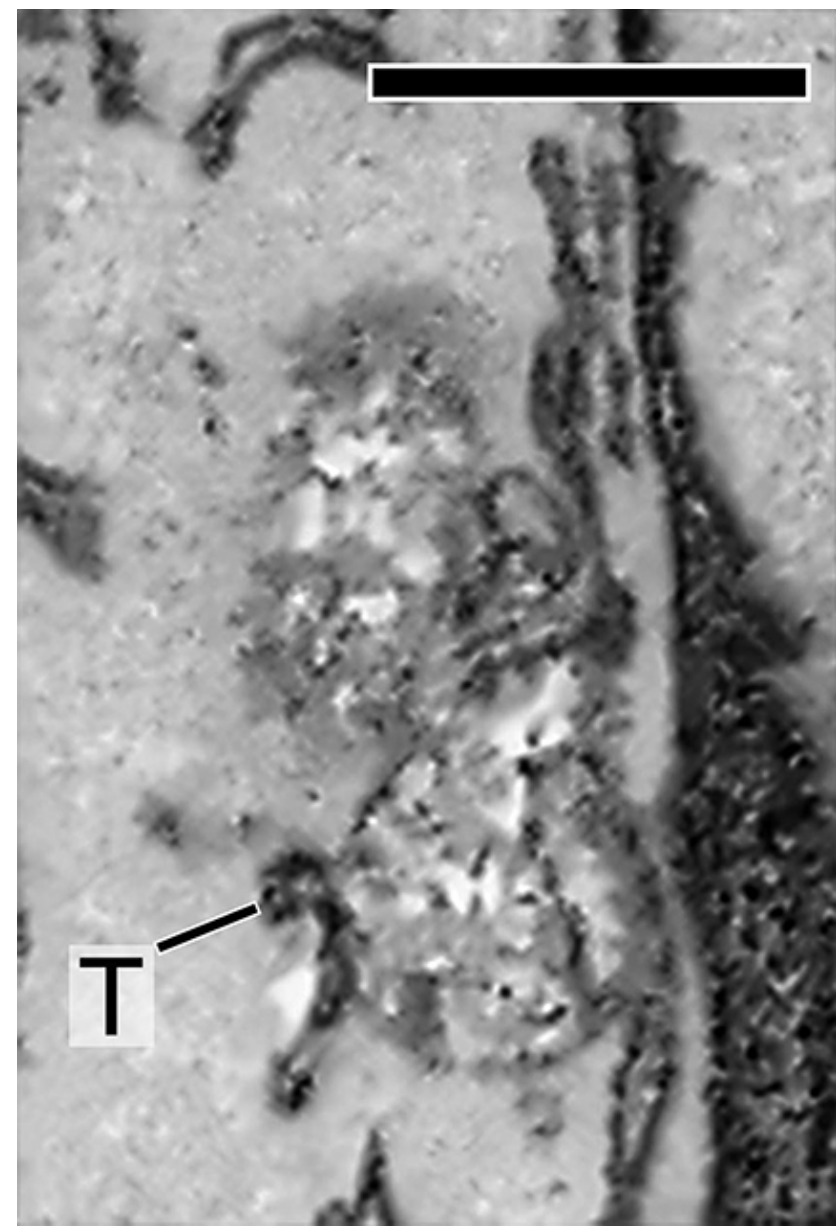

Movie 4. An FIB-SEM movie presents a series of consecutive block face images of a single photoreceptor LE that is highly polymorphic and has multiple contacts with the dark membrane tubules ( $T ; 5 \mathrm{~nm}$ per frame, total 39 frames). $\mathrm{m}$, mitochondria. Scale bar, $500 \mathrm{~nm}$.

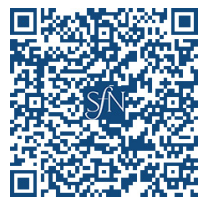

the Lamp1-labeled tubular granular structures in the inner segments/cell bodies (Fig. $7 F, G$ ). The trace amount of PRPH2 in transit was expected to be difficult to detect because the abundant outer segment-expressed PRPH2 could sequester most of the antibodies applied. The nature of the heavily glycosylated LE membrane coats might also impede the antibody accessibility for detecting the LE luminal proteins. To circumvent these technical issues, we set out to visualize the nascent $\mathrm{PRPH} 2$ expressed in the transfected photoreceptors using the $\mathrm{iP}_{\mathrm{CAG}}$-inducible system (Hsu et al., 2015). Specifically, we subjected adult rods, which had been neonatally transfected with $\mathrm{ER}^{\mathrm{T} 2}-\mathrm{Cre}-\mathrm{ER}^{\mathrm{T} 2}$ and floxedSTOP-PRPH2-GFP, to a single dose of 4-hydroxytamoxifen. We showed that PRPH2-GFP expressed for $\geq 1 \mathrm{~d}$ reached the outer segment (Hsu et al., 2015). We then harvested the retinas expressing PRPH2 for $16 \mathrm{~h}$ for immunoEM of GFP. These investigations showed that the high-density gold particles were consistently found in the lumens of the LE-like vacuoles (Fig. $7 H$ ). Though sparse, low-density gold particles were also found on nonmitochondrial, yet-to-be-characterized membrane profiles in the inner segments. No similar signals were seen in sections not stained by primary antibodies. 


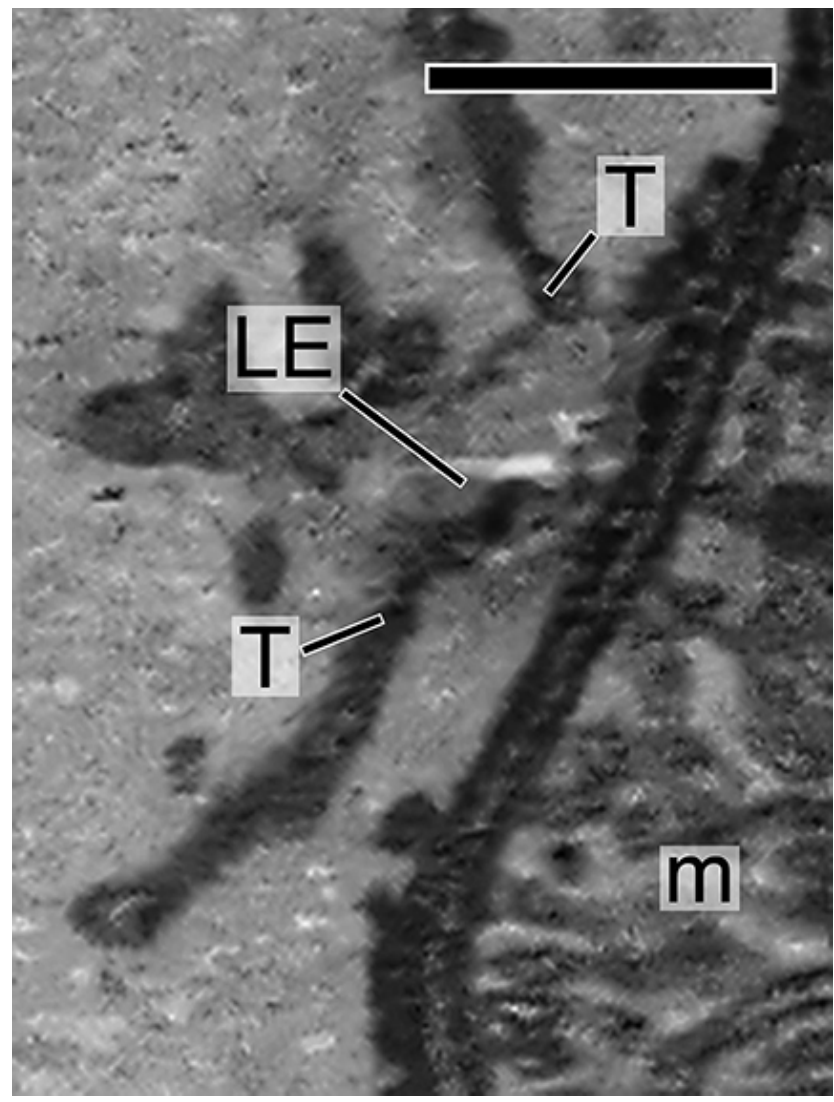

Movie 5. An FIB-SEM movie reveals that a single LE is "intertwined" by dark membrane tubules ( $\mathrm{T} ; 5 \mathrm{~nm}$ per frame, total 28 frames). $\mathrm{m}$, mitochondria. Scale bar, $500 \mathrm{~nm}$.

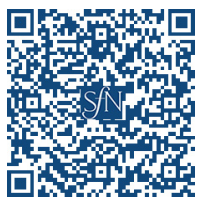

\section{Novel TetOn-inducible expression and cone} transfection method

Thus far, studies of outer segment trafficking have been focused in rods, the predominant photoreceptor cell type in humans and mice. Rods and cones have several distinct features in their cytostructures, including their outer segments. We are keen to address the targeting of PRPH2 in cones because most of the C-terminal PRPH2 mutations, such as 1137TG (Nichols et al., 1993), are linked with cone-dominant macular dystrophies (http://www.retina-international.org/files/sci-news/rdsmut.htm). The current neonatal retinal transfection protocol (used in the above experiments) mainly targets rods. To improve the cone targeting, we transfected the embryonic mouse retinas during the developing stage around the birthdates of the cones in utero (Fig. $8 A$ ). In the retinas harvested from adults, the silhouette outlined by the embryonically transfected GFP showed that a large fraction of transfected retinal cells looked like cones. These cells had a larger synaptic pedicle, a teardrop-shaped cell body, and a conical-shaped outer segment that expressed cone opsins (Fig. 8B).

To enhance the performance and versatility of the temporal transgene regulation in transfected photoreceptors (see Discussion), we established the TetOn-inducible system, which involves a single plasmid encoding of both the reverse CAG-directed TetrtTA3 and Tet operator-miniCMV-directed transgene (Fig. 8C). Doxycycline was used to turn on the expression of the transgene (PRPH2-mCh in this case). We used this method to confirm that in mouse cones the PRPH2-mCh expressed for $4-16 \mathrm{~h}$ was present on the Lamp1-labeled LEs (Fig. 8D) and Rab11b-labeled recycling endosomes (Fig. 8E).

\section{The LE pathway regulates the cone OS targeting of PRPH2}

Subsequently, we used the TetOn system to characterize the outer segment targeting of the $1137 \mathrm{TG}$ PRPH2 in mouse cones. The WT PRPH2-mCh expressed in the mouse cones for $4 \mathrm{~d}$ was predominantly localized in the outer segment (Fig. 9A). While the granular signal of PRPH2 was occasionally seen in the inner segment, almost no signal was ever detected in the cell body/process and synapses (Fig. 9A). In contrast, the 1137TG-mCh expressed in mouse cones for $4 \mathrm{~d}$ was frequently seen in the cell body/process and synapse of transfected cones (Fig. 9A). The predominant outer segment expression of cone opsin was not affected by the expression of either WT or 1137TG mutant PRPH2 (data not shown).

We wished to test the physiological importance of the endosomes in the cone outer segment targeting of PRPH2. To avoid any potential impact on cone development or cone survival, we interfered with the LE or recycling endosome trafficking pathway after the cones matured. We generated plasmids that encode both TetOn-regulated Hrs-shRNA and GFP, and validated their knock-down effect (Fig. 9B). Doxycycline food was used to simultaneously activate the expression of the transfected GFP-HrsshRNA and WT bovine PRPH2 for $4 \mathrm{~d}$. Bovine PRPH2 expressed in cones was detected by the antibody 3B6, which does not crossreact with mouse PRPH2 (Molday et al., 1987). These studies showed that the large majority of the Hrs-suppressed cones had bovine PRPH2 accumulated at the cell body/process and synapse (Fig. 9C). Two independent Hrs-shRNAs, but not the scrambled control shRNA, generated a similar mislocalization index, ruling out the concern of any off-target effect. Furthermore, we showed that the endogenous PRPH2 (detected by anti-mouse PRPH2 antibody $5 \mathrm{H} 2$ ) was also severely mislocalized in cones singly transfected with GFP-Hrs-shRNA (Fig. 9D). Finally, we showed that bovine PRPH2 was also significantly mislocalized in cones that expressed a validated GFP-Rab11b-shRNA for $4 \mathrm{~d}$ (Fig. $9 E, F)$. The Hrs- or Rab11b-suppressed cones look normal and had normal outer segment expression of cone opsins (data not shown), suggesting that the ciliary targeting problem of the PRPH2 was not due to overall cell sickness.

\section{Discussion}

\section{Endosomal pathway underlies the ciliary trafficking of PRPH2}

Until now, ciliary membrane proteins have been known to be transported as post-Golgi vesicles, with or without passing through the recycling endosome, or laterally diffused from the apical plasma membrane (Nachury et al., 2010; Sung and Leroux, 2013). We now add a novel ciliary targeting pathway. Using PRPH2 as a model, we showed that the membrane cargo inside the LE lumen can also be selectively targeted to the cilia, likely through the recycling endosome. PRPH2 mutants that failed to traffic through the LE have impaired ciliary entry. Perturbing the expression or function of the key LE trafficking regulators Rab7 or Hrs inhibited the ciliary localization of PRPH2.

We provided supporting evidence, in both cell cultures and in mouse cones in vivo, demonstrating that the nascent PRPH2 is expressed on the luminal side of the LE. Previous studies showed that the LE luminal membrane proteins (e.g., MHC class II antigen, MMP14) are transported to various membrane compartments on tubules through an incompletely understood mechanism (Kobayashi et al., 2000; Turley et al., 2000; 
A

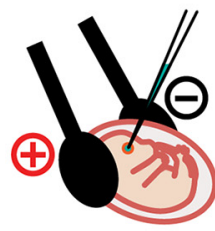

B GFP / Cone opsin / DAPI
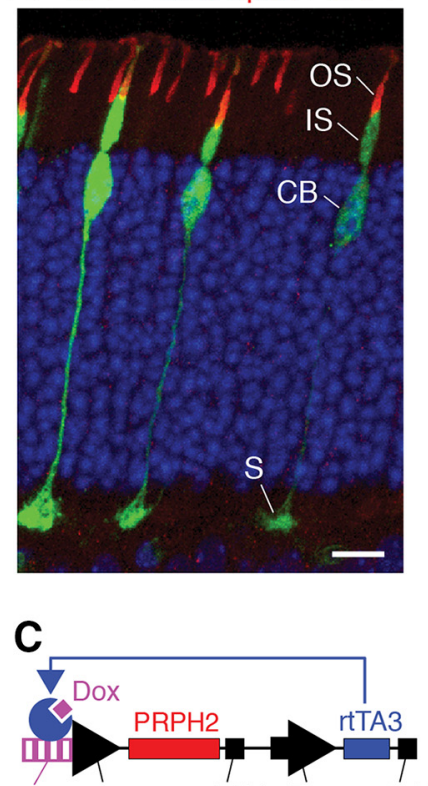

tetO miniCMV poly(A) $P_{C A G} \operatorname{poly}(\mathrm{A})$
D
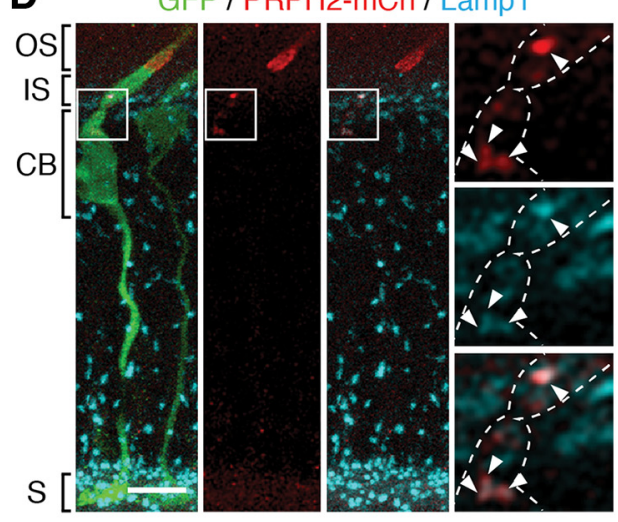

E

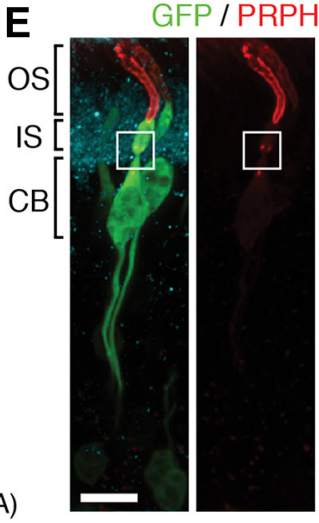

The LE is part of the biosynthetic pathway of PRPH2

Unlike most LE proteins, which are molecules internalized from the cell surface for lysosomal turnover, our study suggests that LE-expressed PRPH2 is not. First, the ER-released PRPH2 is found in the LE without any prior appearance on the plasma membrane or the Golgi. The latter was biochemically supported by the endoglycosidase digestion pattern of PRPH2 (Connell and Molday, 1990; Tian et al., 2014; Zulliger et al., 2015) and the brefeldin A experiments presented in the current article. Although we cannot completely rule out the possibility that a small fraction of PRPH2 enters somewhere else and immediately departs for LEs, our observations strongly indicate that the PRPH2 undertakes a novel ER-to-LE transport pathway that has not been previously described.

Second, the PRPH2 signal is persistently detected in the EGF-negative LEs, indicating that the majority of PRPH2 is not prone to degradation. Third, our EM showed that the photoreceptor-expressed nascent PRPH2 is enriched in the LE vacuoles. Although it is conceivable that some of the LEs serve as a reservoir for the PRPH2 that is going for degradation, the typical-looking lysosomes are rarely seen in mouse photoreceptors. The latter observation makes sense, as outer segment expressed PRPH2 (and other proteins) are mainly digested in the retinal pigment epithelial cells, which engulf the distally shed outer segments (Young, 1967).

\section{Multiple C-terminal sorting signals and} pathways regulate the $\mathrm{LE}$ trafficking of ciliary-directed PRPH2

Although the importance of the cytoplasmic tail of PRPH2 in its ciliary targeting

Kleijmeer et al., 2001; Abrami et al., 2004; Le Blanc et al., 2005). Our time-lapse video recording of MDCK cells detected "red" tubules extending from the PRPH2-mCh-labeled LE granules, and these tubules later broke off and moved away (Movie 6). These observations indicate that PRPH2 leaves LE on tubules too. The 3D EM images of photoreceptors showed that the membrane tubules attached to the LEs are in-and-out of the vesicle-/tubulefilled lumens and belong to the tubular network spanning the entire long axis of the inner segments (Movies 3, 4, 5). We are tempted to speculate that these tubules, which are electron dense due to glycoproteins (Goldfischer et al., 1981), represent the outer segment-directed transport carriers of LE-expressed PRPH2.

The current studies showed that PRPH2 is also expressed on the recycling endosome, and Rab11 functionally regulates its ciliary expression both in vitro and in vivo. Since the newly synthesized PRPH2-mCh-GFP mainly appeared as red (i.e., acidic) granules, the recycling endosomes are likely to be situated "downstream" to the LE trafficking of PRPH2 and serve as an intermediate compartment for the PRPH2 en route to cilium. has been reported, mechanistic insight has been lacking until now. Our systematic analyses of a series of C-terminal mutants suggest that this region of $\mathrm{PRPH} 2$ governs the ciliary targeting by modulating its trafficking through the LE via multiple distinct pathways (Fig. 10A,B). (1) The mutants that lack almost the entire $\mathrm{C}$ terminus (1137TG, $\Delta 53$ ) have concomitant impaired Hrs binding, LE targeting, and ciliary targeting. (2) The C-terminal mutants ( $\Delta 331-342$, V332A) have normal Hrs binding and increased LE accumulation. This indicates that while the distal C-terminal residues (331-342 and V332) are not required for the LE targeting of PRPH2, these residues are required for the LE exit of PRPH2 for the subsequent transport to cilium. Also, piecing the above two pieces of data together, we deduced that the Hrs binding site lies at the proximal C-terminal region of PRPH2 (Fig. 10C). (3) The 4xA mutant, while it binds to Hrs, had impaired ubiquitination and targeting to both LE and cilium. One of the easiest explanations for this result is that C-terminal ubiquitination is required for the LE targeting. Alternatively or additionally, since the C-terminal lysine residues belong to an amphipathic helix structure (Milstein et al., 2017), these lysines might play an Hrs-independent, structural role (e.g., membrane 
A
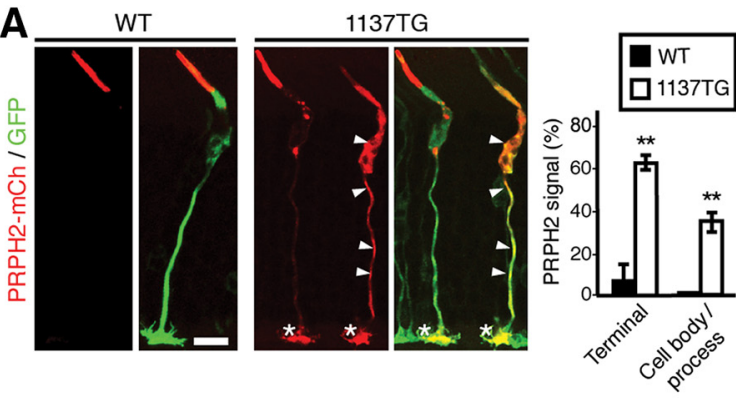

B

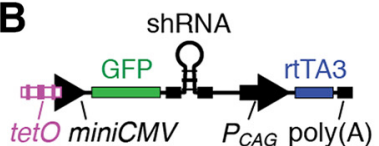

IB: Hrs

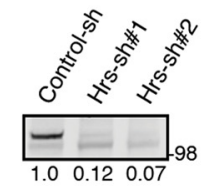

IB: $\beta$-Tublin
D

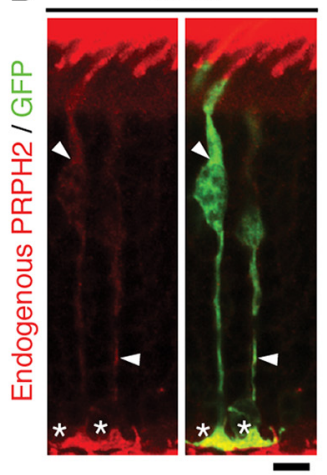

C

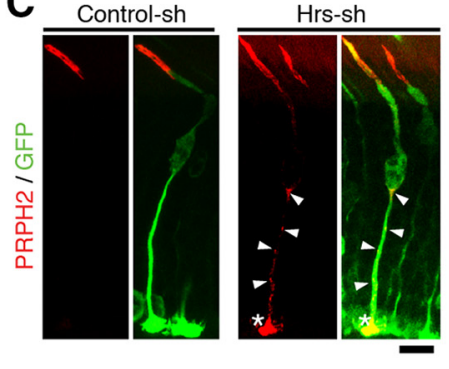

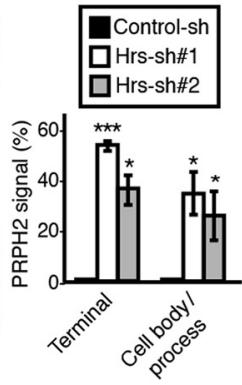

E

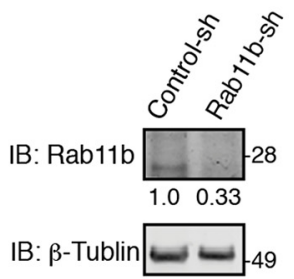

$\mathbf{F}$

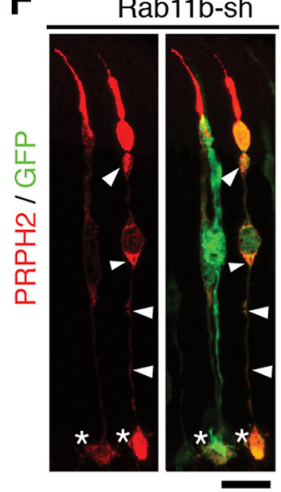

Figure 9. Endosomal trafficking regulates outer segment targeting of PRPH2 in mouse cones. $A$, Representative images of GFP-transfected mouse cones had WT or 1137TG PRPH2-mCh expressed for 4 d. GFP and mCh staining showed that the WT PRPH2 is mainly expressed on the outer segment, while the 1137TG mutant was detectably mislocalized in the cell body/process (arrowheads) and synaptic terminus (asterisks). The percentages of GFP-positive cones that had mislocalized PRPH2-mCh in the indicated cell regions are shown (mean \pm SD; $n=40$ cells, $N=3$ ). Terminal, $p=$ 0.0023; cell body/process, $p=0.0046$. $\boldsymbol{B}, \boldsymbol{E}$, Top in $\boldsymbol{B}$ schematic diagram of Tet0n-GFP-shRNA plasmid. Bottom, Validating the gene-silencing effect of Tet-On-Hrs-shRNA (B) and Tet0n-Rab11shRNA (E). B, Immunoblots of doxycycline-treated 293T cells transfected with GFP-Hrs together scrambled control shRNA or two different Hrs-shRNAs (sh\#1, sh\#2). The relative expression level of GFP-Hrs (internalized by the control protein $\beta$-tubulin) is shown. $E$, Immunoblots containing $661 \mathrm{~W}$ cells transfected with TetOn-regulated GFP-scrambled control shRNA or GFP-Rab11b-shRNA were treated with doxycycline for $3 \mathrm{~d}$, and probed as indicated. The relative Rab11b signals detected (internalized by the $\beta$-tubulin signal) are also shown. $\boldsymbol{C}$, Transfected mouse cones expressing nontagged bovine PRPH2 together with GFP-scrambled control-shRNA or GFP-Hrs-shRNA for $4 \mathrm{~d}$ were stained for bovine PRPH2 (red) and GFP (green). The bovine PRPH2 mislocalized in the cell body/process (arrowheads) and terminus (asterisks) of Hrs-shRNA-transfected cones are prominent. Quantification of the PRPH2 mislocalization in GFP-positive cones is shown (mean \pm SD; $n=$ 34 cells, $N=3$ ). Terminal: Hrs-sh\#1, $p=0.00065$; Hrs-sh\#2, $p=0.0089$; cell body/process: Hrs-sh\#1, $p=0.021$; Hrs-sh\#2, $p=0.042$. D, Two representative mouse cones that had GFP-Hrs-shRNA expressed for $4 \mathrm{~d}$ and were then stained with anti-mouse PRPH2 antibody (red). The endogenous PRPH2 was selectively mislocalized in the cell body/process (arrowheads) and terminus (asterisks) of Hrs-shRNA-transfected cones. $\boldsymbol{F}$, Transfected mouse cones expressing nontagged bovine PRPH2 together with GFP-Rab11-shRNA for $4 \mathrm{~d}$ (via doxycycline food) were stained for bovine PRPH2 (red) and GFP (green). Arrowheads point to the mislocalized bovine PRPH2 at the cell body/process (arrowheads) and synapse (asterisks). ${ }^{* * *} p<0.0005 ;{ }^{* *} p<0.005 ;{ }^{*} p<0.05$, Student's $t$ test. Scale bars: $A, C, D, F, 10 \mu \mathrm{m}$.

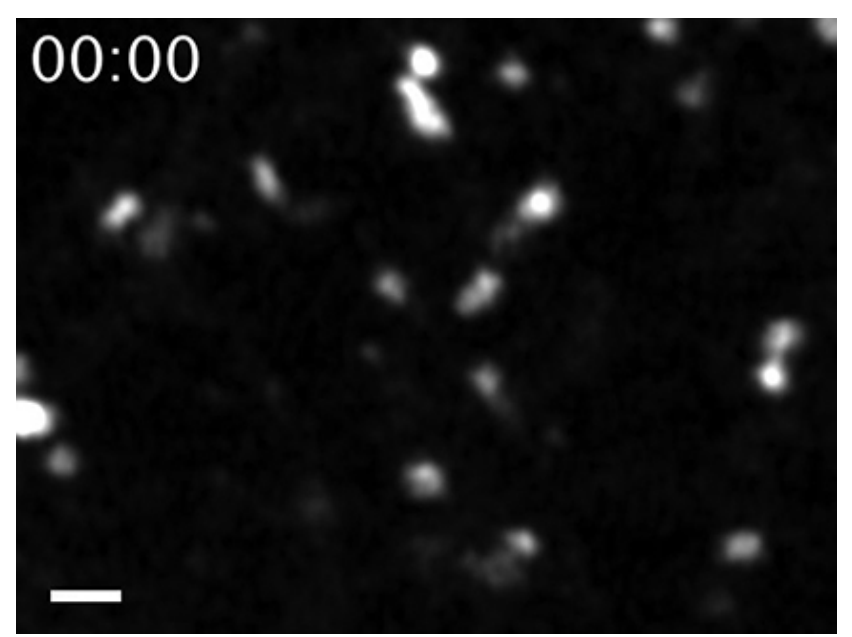

Movie 6. Time-lapse video recording of stably expressed WT PRPH2$\mathrm{mCh}$ in MDCK cells using super resolution-grade Airyscan. Arrows point to a fluorescent tubule that first extends from an LE-like globule and is then pinched off. Scale bar, $1 \mu \mathrm{m}$. curvature formation; Khattree et al., 2013) in LE targeting. In all cases (i.e., $1137 \mathrm{TG}, \Delta 53,4 \mathrm{xA}$ ), the removal of the LE targeting signal from PRPH2 unmasks the "default" Golgi secretory pathway, redirecting the mutants to the lateral side of the cells (in vitro) or the synaptic terminal/cell body/cell process in mouse cones. Like the WT, all tested C-terminal mutants were detected as a single major specie on protein gels (Fig. $5 E$ ), implicating that none of the ciliary targeting defects seen in the C-terminal mutants is due to protein instability and/or aberrant protein aggregation.

The $\mathrm{C}$ terminus of the PRPH2 is a hot spot for human mutations linked to macular/cone dystrophies. The naturally occurring nonsense mutants Q331X, W316X, and Y285X (Felbor et al., 1997; Kohl et al., 1997; Grover et al., 2002), respectively, lack one, two, and all three LE sorting/exit motifs mapped for PRPH2 (Fig. $10 C$ ). These correlations lend clinically relevant support to the LE trafficking pathway of PRPH2.

\section{The physiological significance of LE-expressed PRPH2}

Tetraspanin proteins have a tendency to form membrane microdomains (i.e., tetraspanin web) through homophilic and/or heterophilic protein-protein interactions (Charrin et al., 2014). PRPH2 forms homo-oligomers and hetero-oligomers [with 
A

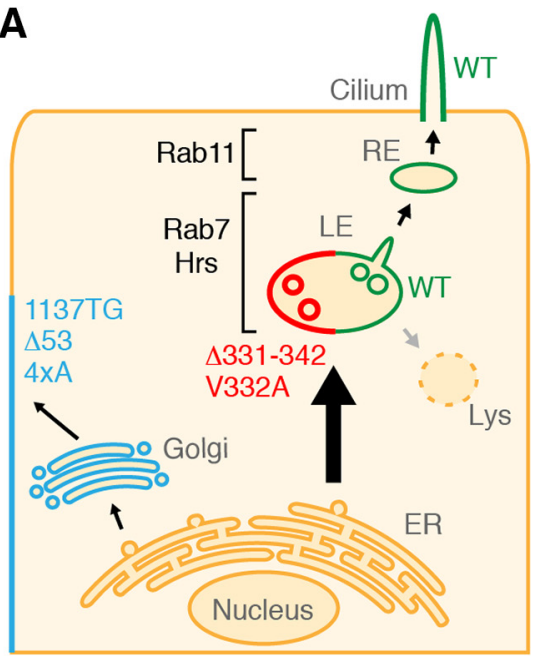

B

\begin{tabular}{|c|c|c|c|}
\hline & $\begin{array}{c}\text { Hrs } \\
\text { binding }\end{array}$ & $\begin{array}{c}\text { LE } \\
\text { targeting }\end{array}$ & $\begin{array}{c}\text { Cilia } \\
\text { targeting }\end{array}$ \\
\hline PRPH2 WT & + & + & + \\
\hline $1137 T G$ & - & - & - \\
\hline$\triangle 53$ & - & - & - \\
\hline $4 \times A$ & + & - & - \\
\hline$\triangle 331-342$ & + & + & - \\
\hline $\mathrm{V332A}$ & + & + & - \\
\hline
\end{tabular}

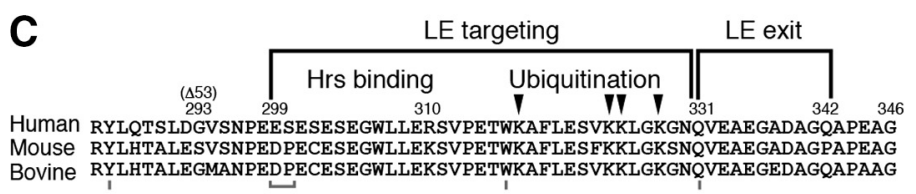

Y285X

1137 TG

W316X

Q331X

Figure 10. Summary of the sorting motifs and mechanism underlies LE and ciliary targeting of PRPH2 and its relevance of retinal dystrophies. $A$, Working model depicting the trafficking route of WT and mutant PRPH2. B, A table shows the phenotypes of various C-terminal mutants. C, The cytoplasmic C-terminal sequences of human, mouse, and bovine PRPH2 and their function motifs and naturally occurring mutation sites are depicted.

ROM1 (retinal outer segment membrane protein 1)], and it is concentrated in the specialized "rim/incisure region" of the rod disc membranes (Molday et al., 1987; Arikawa et al., 1992; Farjo et al., 2006). In agreement with our current finding, several PRPH2-binding proteins are known LE components (e.g., melanoregulin, V-ATPase; Damek-Poprawa et al., 2009; Zulliger et al., 2015). Moreover, an endosomal trafficking regulator Numb has been shown to regulate the outer segment expression of cGMP-gated channel, a protein that interacts with PRPH2 (Couturier et al., 2014; Ramamurthy et al., 2014).

Given that PRPH2 has a pleiotropic role in membrane remodeling and protein interactions (Boesze-Battaglia et al., 1998; Goldberg, 2006; Khattree et al., 2013), its mislocalization could generate spurious changes in membrane structure and cell signaling, contributing to retinal pathology. A growing number of studies demonstrate that the late endocytic compartments are not merely a garbage bin, but rather that these organelles are endowed with wide-ranging roles including autophagy flux, metabolic signaling, lipid homeostasis, and plasma membrane repair (Mukherjee and Maxfield, 2004; Scott et al., 2014; Pu et al., 2016). Our observation that the LE is the major depot for the ciliumdirected PRPH2 in cones opens a new direction for investigating the etiology of retinal dystrophies caused by PRPH2 mutations and brings awareness to the physiological importance of LE biology in mammalian photoreceptors.

\section{Novel cone manipulation techniques}

Our current understanding of cones significantly lags behind our knowledge about rods. It is challenging to detect cone-associated phenotypes in rod-dominant retinas (rods outnumber cones by $\sim 20$-fold in mice and humans). Here, we streamlined a plasmid transfection protocol to perform mouse cone targeting. Additionally, we established the novel TetOn system to perform gainof-function or loss-of-function in transfected photoreceptors. Doxycycline, either by injection or feeding, can be used to activate the TetOn-directed transgenes in a tunable fashion based on the dose of doxycycline. Since PRPH2 is essential for the genesis of the outer segment (Sanyal et al., 1980; Sanyal and Jansen, 1981), it is crucial to investigate the ciliary targeting of the PRPH2 in cones that already have a fully developed outer segment. In the current study, we used a low dose of doxycycline to briefly perturb endosomal trafficking without detectably sickening the cells. We also investigated the distribution of pulseexpressed PRPH2; the low-level PRPH2 fluorescent reporter expression has to be visualized by immunostaining (vs direct fluorescence). Finally, the cell-autonomous phenotypes are easy to identify in transfected cones. This is in contrast with the challenges with phenotyping sometimes seen in Cre-loxmediated conditional knock-out mice due to mosaicism or hypomorphism. Thus, the cone manipulation described here will expedite discovery for cone biology and the etiology of a high number of cone/macular dystrophies, which remain largely unexplored.

\section{References}

Abrami L, Lindsay M, Parton RG, Leppla SH, van der Goot FG (2004) Membrane insertion of anthrax protective antigen and cytoplasmic delivery of lethal factor occur at different stages of the endocytic pathway. J Cell Biol 166:645-651.

Al-Ubaidi MR, Matsumoto H, Kurono S, Singh A (2008) Proteomics profiling of the cone photoreceptor cell line, 661W. Adv Exp Med Biol 613:301-311.

Arikawa K, Molday LL, Molday RS, Williams DS (1992) Localization of peripherin/rds in the disk membranes of cone and rod photoreceptors: relationship to disk membrane morphogenesis and retinal degeneration. J Cell Biol 116:659-667.

Aronson DE, Costantini LM, Snapp EL (2011) Superfolder GFP is fluorescent in oxidizing environments when targeted via the sec translocon. Traffic 12:543-548.

Boesze-Battaglia K, Lamba OP, Napoli AA Jr, Sinha S, Guo Y (1998) Fusion between retinal rod outer segment membranes and model membranes: a role for photoreceptor peripherin/rds. Biochemistry 37:9477-9487.

Bryant DM, Datta A, Rodríguez-Fraticelli AE, Peränen J, Martín-Belmonte F, Mostov KE (2010) A molecular network for de novo generation of the apical surface and lumen. Nat Cell Biol 12:1035-1045.

Chang K, Elledge SJ, Hannon GJ (2006) Lessons from nature: microRNAbased shRNA libraries. Nat Methods 3:707-714.

Charrin S, Jouannet S, Boucheix C, Rubinstein E (2014) Tetraspanins at a glance. J Cell Sci 127:3641-3648.

Chuang JZ, Zhao Y, Sung CH (2007) SARA-regulated vesicular targeting underlies formation of the light-sensing organelle in mammalian rods. Cell 130:535-547.

Chuang JZ, Hsu YC, Sung CH (2015) Ultrastructural visualization of transciliary rhodopsin cargoes in mammalian rods. Cilia 4:4. 
Cole NB, Smith CL, Sciaky N, Terasaki M, Edidin M, Lippincott-Schwartz J (1996) Diffusional mobility of Golgi proteins in membranes of living cells. Science 273:797-801.

Conley SM, Stricker HM, Naash MI (2010) Biochemical analysis of phenotypic diversity associated with mutations in codon 244 of the retinal degeneration slow gene. Biochemistry 49:905-911.

Connell GJ, Molday RS (1990) Molecular cloning, primary structure, and orientation of the vertebrate photoreceptor cell protein peripherin in the rod outer segment disk membrane. Biochemistry 29:4691-4698.

Connell G, Bascom R, Molday L, Reid D, McInnes RR, Molday RS (1991) Photoreceptor peripherin is the normal product of the gene responsible for retinal degeneration in the rds mouse. Proc Natl Acad Sci U S A $88: 723-726$.

Couturier L, Trylinski M, Mazouni K, Darnet L, Schweisguth F (2014) A fluorescent tagging approach in drosophila reveals late endosomal trafficking of notch and sanpodo. J Cell Biol 207:351-363.

Damek-Poprawa M, Diemer T, Lopes VS, Lillo C, Harper DC, Marks MS, Wu Y, Sparrow JR, Rachel RA, Williams DS, Boesze-Battaglia K (2009) Melanoregulin (MREG) modulates lysosome function in pigment epithelial cells. J Biol Chem 284:10877-10889.

Eden ER, Burgoyne T, Edgar JR, Sorkin A, Futter CE (2012) The relationship between ER-multivesicular body membrane contacts and the ESCRT machinery. Biochem Soc Trans 40:464-468.

Edgar JR, Eden ER, Futter CE (2014) Hrs- and CD63-dependent competing mechanisms make different sized endosomal intraluminal vesicles. Traffic 15:197-211.

Falcón-Pérez JM, Nazarian R, Sabatti C, Dell'Angelica EC (2005) Distribution and dynamics of Lampl-containing endocytic organelles in fibroblasts deficient in BLOC-3. J Cell Sci 118:5243-5255.

Farjo R, Skaggs JS, Nagel BA, Quiambao AB, Nash ZA, Fliesler SJ, Naash MI (2006) Retention of function without normal disc morphogenesis occurs in cone but not rod photoreceptors. J Cell Biol 173:59-68.

Felbor U, Schilling H, Weber BH (1997) Adult vitelliform macular dystrophy is frequently associated with mutations in the peripherin/RDS gene. Hum Mutat 10:301-309.

Felder S, Miller K, Moehren G, Ullrich A, Schlessinger J, Hopkins CR (1990) Kinase activity controls the sorting of the epidermal growth factor receptor within the multivesicular body. Cell 61:623-634.

Friedman JR, Dibenedetto JR, West M, Rowland AA, Voeltz GK (2013) Endoplasmic reticulum-endosome contact increases as endosomes traffic and mature. Mol Biol Cell 24:1030-1040.

Gibson A, Futter CE, Maxwell S, Allchin EH, Shipman M, Kraehenbuhl JP, Domingo D, Odorizzi G, Trowbridge IS, Hopkins CR (1998) Sorting mechanisms regulating membrane protein traffic in the apical transcytotic pathway of polarized MDCK cells. J Cell Biol 143:81-94.

Goldberg AF (2006) Role of peripherin/rds in vertebrate photoreceptor architecture and inherited retinal degenerations. Int Rev Cytol 253:131175.

Goldberg AF, Moritz OL, Molday RS (1995) Heterologous expression of photoreceptor peripherin/rds and rom-1 in COS-1 cells: assembly, interactions, and localization of multisubunit complexes. Biochemistry 34: 14213-14219.

Goldfischer S, Kress Y, Coltoff-Schiller B, Berman J (1981) Primary fixation in osmium-potassium ferrocyanide: the staining of glycogen, glycoproteins, elastin, an intranuclear reticular structure, and intercisternal trabeculae. J Histochem Cytochem 29:1105-1111.

Gravotta D, Carvajal-Gonzalez JM, Mattera R, Deborde S, Banfelder JR, Bonifacino JS, Rodriguez-Boulan E (2012) The clathrin adaptor AP-1A mediates basolateral polarity. Dev Cell 22:811-823.

Grover S, Fishman GA, Stone EM (2002) Atypical presentation of pattern dystrophy in two families with peripherin/RDS mutations. Ophthalmology 109:1110-1117.

Hashimoto Y, Fukasawa M, Kuniyasu H, Yagi K, Kondoh M (2017) Claudin-targeted drug development using anti-claudin monoclonal antibodies to treat hepatitis and cancer. Ann N Y Acad Sci 1397:5-16.

Hsu YC, Chuang JZ, Sung CH (2015) Light regulates the ciliary protein transport and outer segment disc renewal of mammalian photoreceptors. Dev Cell 32:731-742.

Jou TS, Nelson WJ (1998) Effects of regulated expression of mutant RhoA and Racl small GTPases on the development of epithelial (MDCK) cell polarity. J Cell Biol 142:85-100.

Kajimoto T, Okada T, Miya S, Zhang L, Nakamura S (2013) Ongoing acti- vation of sphingosine 1-phosphate receptors mediates maturation of exosomal multivesicular endosomes. Nat Commun 4:2712.

Khattree N, Ritter LM, Goldberg AF (2013) Membrane curvature generation by a C-terminal amphipathic helix in peripherin-2/rds, a tetraspanin required for photoreceptor sensory cilium morphogenesis. J Cell Sci 126:4659-4670.

Kleijmeer M, Ramm G, Schuurhuis D, Griffith J, Rescigno M, RicciardiCastagnoli P, Rudensky AY, Ossendorp F, Melief CJ, Stoorvogel W, Geuze HJ (2001) Reorganization of multivesicular bodies regulates MHC class II antigen presentation by dendritic cells. J Cell Biol 155:53-63.

Kobayashi T, Vischer UM, Rosnoblet C, Lebrand C, Lindsay M, Parton RG, Kruithof EK, Gruenberg J (2000) The tetraspanin CD63/lamp3 cycles between endocytic and secretory compartments in human endothelial cells. Mol Biol Cell 11:1829-1843.

Kobayashi T, Beuchat MH, Chevallier J, Makino A, Mayran N, Escola JM, Lebrand C, Cosson P, Kobayashi T, Gruenberg J (2002) Separation and characterization of late endosomal membrane domains. J Biol Chem 277:32157-32164.

Kohl S, Christ-Adler M, Apfelstedt-Sylla E, Kellner U, Eckstein A, Zrenner E, Wissinger B (1997) RDS/peripherin gene mutations are frequent causes of central retinal dystrophies. J Med Genet 34:620-626.

Lawe DC, Patki V, Heller-Harrison R, Lambright D, Corvera S (2000) The FYVE domain of early endosome antigen 1 is required for both phosphatidylinositol 3-phosphate and Rab5 binding. critical role of this dual interaction for endosomal localization. J Biol Chem 275:3699-3705.

Le Blanc I, Luyet PP, Pons V, Ferguson C, Emans N, Petiot A, Mayran N, Demaurex N, Fauré J, Sadoul R, Parton RG, Gruenberg J (2005) Endosome-to-cytosol transport of viral nucleocapsids. Nat Cell Biol 7:653-664

Loewen CJ, Moritz OL, Tam BM, Papermaster DS, Molday RS (2003) The role of subunit assembly in peripherin-2 targeting to rod photoreceptor disk membranes and retinitis pigmentosa. Mol Biol Cell 14:3400-3413.

Matsuda T, Cepko CL (2007) Controlled expression of transgenes introduced by in vivo electroporation. Proc Natl Acad Sci U S A 104:10271032.

Milstein ML, Kimler VA, Ghatak C, Ladokhin AS, Goldberg AFX (2017) An inducible amphipathic helix within the intrinsically disordered C terminus can participate in membrane curvature generation by peripherin-2/ rds. J Biol Chem 292:7850-7865.

Mittelbrunn M, Gutiérrez-Vázque C, Villarroya-Beltri C, González S, Sánchez-Cabo F, González MÁ, Bernad A, Sánchez-Madrid F (2011) Unidirectional transfer of microRNA-loaded exosomes from $\mathrm{T}$ cells to antigen-presenting cells. Nat Commun 2:282.

Molday RS, Hicks D, Molday L (1987) Peripherin. A rim-specific membrane protein of rod outer segment discs. Invest Ophthalmol Vis Sci 28:50-61.

Molday RS, Molday LL, Loewen CJ (2004) Role of subunit assembly in autosomal dominant retinitis pigmentosa linked to mutations in peripherin 2. Novartis Found Symp 255:95-112.

Mukherjee S, Maxfield FR (2004) Lipid and cholesterol trafficking in NPC. Biochim Biophys Acta 1685:28-37.

Nabi IR, Le Bivic A, Fambrough D, Rodriguez-Boulan E (1991) An endogenous MDCK lysosomal membrane glycoprotein is targeted basolaterally before delivery to lysosomes. J Cell Biol 115:1573-1584.

Nachury MV, Seeley ES, Jin H (2010) Trafficking to the ciliary membrane: how to get across the periciliary diffusion barrier? Annu Rev Cell Dev Biol 26:59-87.

Nichols BE, Drack AV, Vandenburgh K, Kimura AE, Sheffield VC, Stone EM (1993) A 2 base pair deletion in the RDS gene associated with butterflyshaped pigment dystrophy of the fovea. Hum Mol Genet 2:1347.

Pédelacq JD, Cabantous S, Tran T, Terwilliger TC, Waldo GS (2006) Engineering and characterization of a superfolder green fluorescent protein. Nat Biotechnol 24:79-88.

Piper RC, Katzmann DJ (2007) Biogenesis and function of multivesicular bodies. Annu Rev Cell Dev Biol 23:519-547.

Pu J, Guardia CM, Keren-Kaplan T, Bonifacino JS (2016) Mechanisms and functions of lysosome positioning. J Cell Sci 129:4329-4339.

Raiborg C, Stenmark H (2002) Hrs and endocytic sorting of ubiquitinated membrane proteins. Cell Struct Funct 27:403-408.

Ramamurthy V, Jolicoeur C, Koutroumbas D, Mühlhans J, Le YZ, Hauswirth WW, Giessl A, Cayouette M (2014) Numb regulates the polarized deliv- 
ery of cyclic nucleotide-gated ion channels in rod photoreceptor cilia. J Neurosci 34:13976-13987.

Salinas RY, Baker SA, Gospe SM 3rd, Arshavsky VY (2013) A single valine residue plays an essential role in peripherin/rds targeting to photoreceptor outer segments. PLoS One 8:e54292.

Salinas RY, Pearring JN, Ding JD, Spencer WJ, Hao Y, Arshavsky VY (2017) Photoreceptor discs form through peripherin-dependent suppression of ciliary ectosome release. J Cell Biol 216:1489-1499.

Sanyal S, Jansen HG (1981) Absence of receptor segments in the retina of rds mutant mice. Neurosci Lett 21:23-26.

Sanyal S, De Ruiter A, Hawkins RK (1980) Development and degeneration of retina in rds mutant mice: light microscopy. J Comp Neurol 194:193-207.

Scott CC, Vacca F, Gruenberg J (2014) Endosome maturation, transport and functions. Semin Cell Dev Biol 31:2-10.

Stuck MW, Conley SM, Naash MI (2016) PRPH2/RDS and ROM-1: historical context, current views and future considerations. Prog Retin Eye Res $52: 47-63$.

Sung CH, Leroux MR (2013) The roles of evolutionarily conserved functional modules in cilia-related trafficking. Nat Cell Biol 15:1387-1397.

Taelman VF, Dobrowolski R, Plouhinec JL, Fuentealba LC, Vorwald PP, Gumper I, Sabatini DD, De Robertis EM (2010) Wnt signaling requires sequestration of glycogen synthase kinase 3 inside multivesicular endosomes. Cell 143:1136-1148.

Tam BM, Moritz OL, Papermaster DS (2004) The C terminus of peripherin/ rds participates in rod outer segment targeting and alignment of disk incisures. Mol Biol Cell 15:2027-2037.

Tan E, Ding XQ, Saadi A, Agarwal N, Naash MI, Al-Ubaidi MR (2004) Expression of cone-photoreceptor-specific antigens in a cell line derived from retinal tumors in transgenic mice. Invest Ophthalmol Vis Sci $45: 764-768$.
Thuenauer R, Hsu YC, Carvajal-Gonzalez JM, Deborde S, Chuang JZ, Römer W, Sonnleitner A, Rodriguez-Boulan E, Sung CH (2014) Fourdimensional live imaging of apical biosynthetic trafficking reveals a postGolgi sorting role of apical endosomal intermediates. Proc Natl Acad Sci U S A 111:4127-4132.

Tian G, Ropelewski P, Nemet I, Lee R, Lodowski KH, Imanishi Y (2014) An unconventional secretory pathway mediates the cilia targeting of peripherin/rds. J Neurosci 34:992-1006.

Tooze J, Hollinshead M, Ludwig T, Howell K, Hoflack B, Kern H (1990) In exocrine pancreas, the basolateral endocytic pathway converges with the autophagic pathway immediately after the early endosome. J Cell Biol 111:329-345.

Turley SJ, Inaba K, Garrett WS, Ebersold M, Unternaehrer J, Steinman RM, Mellman I (2000) Transport of peptide-MHC class II complexes in developing dendritic cells. Science 288:522-527.

Urbé S, Sachse M, Row PE, Preisinger C, Barr FA, Strous G, Klumperman J, Clague MJ (2003) The UIM domain of hrs couples receptor sorting to vesicle formation. J Cell Sci 116:4169-4179.

Wang Y, Macke JP, Merbs SL, Zack DJ, Klaunberg B, Bennett J, Gearhart J, Nathans J (1992) A locus control region adjacent to the human red and green visual pigment genes. Neuron 9:429-440.

White IJ, Bailey LM, Aghakhani MR, Moss SE, Futter CE (2006) EGF stimulates annexin 1-dependent inward vesiculation in a multivesicular endosome subpopulation. EMBO J 25:1-12.

Young RW (1967) The renewal of photoreceptor cell outer segments. J Cell Biol 33:61-72.

Zulliger R, Conley SM, Mwoyosvi ML, Stuck MW, Azadi S, Naash MI (2015) SNAREs interact with retinal degeneration slow and rod outer segment membrane protein-1 during conventional and unconventional outer segment targeting. PLoS One 10:e0138508. 